\title{
DISCREPANCY BOUNDS FOR THE DISTRIBUTION OF THE RIEMANN ZETA-FUNCTION AND APPLICATIONS
}

\author{
YOUNESS LAMZOURI, STEPHEN LESTER, AND MAKSYM RADZIWIŁE
}

\begin{abstract}
We investigate the distribution of the Riemann zeta-function on the line $\operatorname{Re}(s)=\sigma$. For $\frac{1}{2}<\sigma \leq 1$ we obtain an upper bound on the discrepancy between the distribution of $\zeta(s)$ and that of its random model, improving results of Harman and Matsumoto. Additionally, we examine the distribution of the extreme values of $\zeta(s)$ inside of the critical strip, strengthening a previous result of the first author.

As an application of these results we obtain the first effective error term for the number of solutions to $\zeta(s)=a$ in a strip $\frac{1}{2}<\sigma_{1}<\sigma_{2}<1$. Previously in the strip $\frac{1}{2}<\sigma<1$ only an asymptotic estimate was available due to a result of Borchsenius and Jessen from 1948 and effective estimates were known only slightly to the left of the half-line, under the Riemann hypothesis (due to Selberg) and to the right of the abscissa of absolute convergence (due to Matsumoto). In general our results are an improvement of the classical Bohr-Jessen framework and are also applicable to counting the zeros of the Epstein zeta-function.
\end{abstract}

\section{INTRODUCTION AND STATEMENT OF MAIN RESULTS}

Let $\{X(p)\}_{p}$ be a sequence of independent random variables uniformly distributed on the unit circle where $p$ runs over the prime numbers. Consider the random Euler product

$$
\zeta(\sigma, X)=\prod_{p}\left(1-\frac{X(p)}{p^{\sigma}}\right)^{-1}
$$

which converges almost surely for $\sigma>\frac{1}{2}$. Due to the unique factorization of the integers we intuitively expect that the functions $p^{-i t}$ interact like the independent random variables $X(p)$. This suggests that $\zeta(\sigma, X)$ should be a good model for the Riemann zeta-function, and one may ask: How well does the distribution of $\zeta(\sigma, X)$ approximate that of the Riemann zeta-function?

A theorem of Bohr and Jessen [1] asserts that $\log \zeta(\sigma+i t)$ has a continuous limiting distribution in the complex plane for $\sigma>\frac{1}{2}$. In fact, it can be seen from their work that $\log \zeta(\sigma+i t)$ converges in distribution to $\log \zeta(\sigma, X)$ for $\sigma>\frac{1}{2}$. In this article we investigate the discrepancy between the distributions of the random variable $\log \zeta(\sigma, X)$

2010 Mathematics Subject Classification. Primary 11M06.

The first author is supported in part by an NSERC Discovery grant. The third author is partially supported by NSF grant DMS-1128155. The research leading to these results has received funding from the European Research Council under the European Union's Seventh Framework Programme (FP7/2007-2013) / ERC grant agreement $\mathrm{n}^{\circ} 320755$. 
and that of $\log \zeta(\sigma+i t)$, i.e.

$$
D_{\sigma}(T):=\sup _{\mathcal{R}}\left|\mathbb{P}_{T}(\log \zeta(\sigma+i t) \in \mathcal{R})-\mathbb{P}(\log \zeta(\sigma, X) \in \mathcal{R})\right|,
$$

where the supremum is taken over rectangles $\mathcal{R}$ with sides parallel to the coordinate axes and where

$$
\mathbb{P}_{T}(f(t) \in \mathcal{R}):=\frac{1}{T} \cdot \operatorname{meas}\{T \leq t \leq 2 T: f(t) \in \mathcal{R}\} .
$$

This quantity measures the extent to which the distribution function of the random variable $\log \zeta(\sigma, X)$ approximates that of $\log \zeta(\sigma+i t)$. We prove

Theorem 1.1. Let $\frac{1}{2}<\sigma<1$ be fixed. Then

$$
D_{\sigma}(T) \ll \frac{1}{(\log T)^{\sigma}} .
$$

Additionally, for $\sigma=1$ we have

$$
D_{1}(T) \ll \frac{\log \log T}{\log T} .
$$

Theorem 1.1 improves upon a previous discrepancy estimate due to G. Harman and K. Matsumoto [6]. For fixed $\frac{1}{2}<\sigma \leq 1$ they showed that the discrepancy satisfies the bound

$$
D_{\sigma}(T) \ll \frac{1}{(\log T)^{(4 \sigma-2) /(21+8 \sigma)-\varepsilon}}
$$

for any $\varepsilon>0$. One new feature of our estimate is that the power of the logarithm does not decay to zero as $\sigma \rightarrow \frac{1}{2}$. We introduce a different technique to study this problem that relies upon careful analysis of large complex moments of the Riemann zeta-function inside of the critical strip. Some of the tools developed by A. Selberg to study the distribution of $\log \zeta\left(\frac{1}{2}+i t\right)$ are also used, such as Beurling-Selberg functions.

An important problem in the theory of the Riemann zeta-function is to understand its maximal order within the critical strip. The Riemann hypothesis implies that for $\frac{1}{2}<\sigma<1$ and $t$ large we have $\log |\zeta(\sigma+i t)| \ll(\log t)^{2-2 \sigma+o(1)}$ (see Theorem 14.5 of [16]). On the other hand, Montgomery [14] proved that $\log |\zeta(\sigma+i t)|=\Omega\left((\log t)^{1-\sigma+o(1)}\right)$, and based on a probabilistic argument, he conjectured that this omega result is in fact optimal, namely that $\log |\zeta(\sigma+i t)| \ll(\log t)^{1-\sigma+o(1)}$. This motivates the study of the extent to which the extreme values of $\zeta(\sigma+i t)$ can be modeled by those of the random variable $\zeta(\sigma, X)$. For if the distribution of the extreme values of $\zeta(\sigma+i t)$ matches that of $\zeta(\sigma, X)$ in the viable range then Montgomery's conjecture follows.

In 9] the first author obtained an asymptotic estimate for $\log \mathbb{P}_{T}(\log |\zeta(\sigma+i t)|>\tau)$ in nearly the full conjectured range of $\tau$. More precisely, he showed that there is a positive constant $A(\sigma)$, such that uniformly in the range $\tau \ll(\log T)^{1-\sigma+o(1)}$, we have

$$
\begin{aligned}
\log \mathbb{P}_{T}(\log |\zeta(\sigma+i t)|>\tau) & =(1+o(1)) \log \mathbb{P}(\log |\zeta(\sigma, X)|>\tau) \\
& =(-A(\sigma)+o(1)) \tau^{\frac{1}{1-\sigma}}(\log \tau)^{\frac{\sigma}{1-\sigma}}
\end{aligned}
$$


We strengthen this result, obtaining an asymptotic formula for $\mathbb{P}_{T}(\log |\zeta(\sigma+i t)|>\tau)$ in the same range.

Theorem 1.2. Let $\frac{1}{2}<\sigma<1$ be fixed. There exists a constant $b(\sigma)>0$ such that for $3 \leq \tau \leq b(\sigma)(\log T)^{1-\sigma}(\log \log T)^{1-\frac{1}{\sigma}}$ we have

$\mathbb{P}_{T}(\log |\zeta(\sigma+i t)|>\tau)=\mathbb{P}(\log |\zeta(\sigma, X)|>\tau) \times\left(1+O\left(\frac{(\tau \log \tau)^{\frac{\sigma}{1-\sigma}} \cdot(\log \log T)}{(\log T)^{\sigma}}\right)\right)$.

Moreover, the same asymptotic estimate holds when $\log |\zeta(\sigma+i t)|$ and $\log |\zeta(\sigma, X)|$ are replaced by $\arg \zeta(\sigma+i t)$ and $\arg \zeta(\sigma, X)$ respectively.

The terms $(\log T)^{\sigma}$ appearing in the error term in Theorem 1.2 and in Theorem 1.1 are related. An improvement in our method would produce an improvement in both results. Since we do not believe that we will be able to extend significantly the range of Theorem 1.2, it seems that our bound for $D_{\sigma}(T)$ is as well optimal given the method used.

We also apply Theorem 1.1 to study the roots, $s$, to the equation $\zeta(s)=a$ where $a$ is a nonzero complex number. These points are known as $a$-points and the study of their distribution is a classical topic in the theory of the Riemann zeta-function.

Let $N_{a}\left(\sigma_{1}, \sigma_{2} ; T\right)$ be the number of $a$-points in the strip $\frac{1}{2}<\sigma_{1}<\sigma<\sigma_{2}<1$, $T \leq t \leq 2 T$. In 1948 Borchsenius and Jessen 3 proved that there exists a constant $c\left(a, \sigma_{1}, \sigma_{2}\right)>0$ such that as $T \rightarrow \infty$

$$
N_{a}\left(\sigma_{1}, \sigma_{2} ; T\right) \sim c\left(a, \sigma_{1}, \sigma_{2}\right) T .
$$

The constant $c\left(a, \sigma_{1}, \sigma_{2}\right)$ can be explicitly given in terms of the random variable $\zeta(\sigma, X)$. Indeed, let

Then,

$$
f_{a}(\sigma)=\mathbb{E}(\log |\zeta(\sigma, X)-a|)
$$

$$
c\left(a, \sigma_{1}, \sigma_{2}\right)=\frac{f_{a}^{\prime}\left(\sigma_{2}\right)-f_{a}^{\prime}\left(\sigma_{1}\right)}{2 \pi} .
$$

The differentiability of $f_{a}(\sigma)$ is not trivial, and was established by Borchsenius and Jessen.

Using Theorem 1.1 we obtain the first effective error term for $N_{a}\left(\sigma_{1}, \sigma_{2} ; T\right)$ valid for $\sigma_{1}<\sigma_{2}$ in the critical strip.

Theorem 1.3. Let $\frac{1}{2}<\sigma_{1}<\sigma_{2}<1$. For every nonzero complex number a there exists a constant $c\left(a, \sigma_{1}, \sigma_{2}\right)>0$ such that

$$
N_{a}\left(\sigma_{1}, \sigma_{2} ; T\right)=c\left(a, \sigma_{1}, \sigma_{2}\right) T+O\left(T \cdot \frac{\log \log T}{(\log T)^{\sigma_{1} / 2}}\right) .
$$

Inside the critical strip, an effective error term was known previously only slightly to the left of the half-line under the assumption of the Riemann hypothesis, thanks to unpublished work of Selberg (see [17], Chapter 8). In the region of absolute convergence $(\sigma>1)$, Matsumoto [12, [13, with some additional constraints, has given a formula for the number of $a$-points of $\log \zeta(s)$ with an error term that has a power saving of 
$\log \log T$. We have not determined the limits of our method for $\sigma>1$, but it should give a formula for the number of $a$-points of $\zeta(s)$ (and $\log \zeta(s)$ as well) with an error term with a saving of at least $(\log T)^{1 / 2}$.

The error term in Theorem 1.3 is essentially the square-root of the discrepancy $D_{\sigma}(T)$. We have not been able to determine conjecturally the correct size of $D_{\sigma}(T)$. In this direction we have only the following remark.

Remark 1.1. We have,

$$
D_{\sigma}(T)=\Omega\left(T^{1-2 \sigma-\varepsilon}\right) .
$$

Moreover, If $D_{\sigma}(T)=O\left(T^{1-2 \sigma+\varepsilon}\right)$ then the Zero Density Hypothesis holds.

We give a proof of this remark in the Appendix. There is an apparent discrepancy between our lower and upper bound for $D_{\sigma}(T)$. It would be very interesting to work out a reliable heuristic to predict the correct size of $D_{\sigma}(T)$.

It is likely that our ideas can be generalized to other situations where the BohrJessen framework applies [1. For example, our method should adapt to the study of the zeros of the Epstein zeta-function of a quadratic form with class number $n$. We expect the method to show that the number of zeros of the Epstein zeta-function in the strip $\sigma_{1}<\sigma<\sigma_{2}$ is $c\left(\sigma_{1}, \sigma_{2}\right) T+O\left(T(\log T)^{-A\left(\sigma_{1}, n\right)}\right)$ with an $A\left(\sigma_{1}, n\right) \rightarrow 0$ as $n \rightarrow \infty$. This would refine previous results of Bombieri and Mueller [2] and Lee's improvement there-of [11].

\section{Key iDEAS AND DETAILED RESUlts}

In probability theory, the classical Berry-Esseen Theorem states that if the characteristic functions of two real valued random variables are close, then their corresponding probability distributions are close as well. In order to establish Theorem 1.1 the key ingredient is to show that the characteristic function of the joint distribution of $\operatorname{Re} \log \zeta(\sigma+i t)$ and $\operatorname{Im} \log \zeta(\sigma+i t)$ can be very well approximated by the corresponding characteristic function of the random model $\log \zeta(\sigma, X)$. For $u, v \in \mathbb{R}$ we define

$$
\Phi_{\sigma, T}(u, v):=\frac{1}{T} \int_{T}^{2 T} \exp (i u \operatorname{Re} \log \zeta(\sigma+i t)+i v \operatorname{Im} \log \zeta(\sigma+i t)) d t
$$

and

$$
\Phi_{\sigma}^{\mathrm{rand}}(u, v):=\mathbb{E}(\exp (i u \operatorname{Re} \log \zeta(\sigma, X)+i v \operatorname{Im} \log \zeta(\sigma, X))) .
$$

Then we prove

Theorem 2.1. Let $\frac{1}{2}<\sigma<1$ and $A \geq 1$ be fixed. There exists a constant $b_{1}=b_{1}(\sigma, A)$ such that for $|u|,|v| \leq b_{1}(\log T)^{\sigma}$ we have

$$
\Phi_{\sigma, T}(u, v)=\Phi_{\sigma}^{\mathrm{rand}}(u, v)+O\left(\frac{1}{(\log T)^{A}}\right) .
$$

Moreover, there exists a constant $b_{2}=b_{2}(A)$ such that for $|u|,|v| \leq b_{2} \log T / \log \log T$ we have

$$
\Phi_{1, T}(u, v)=\Phi_{1}^{\mathrm{rand}}(u, v)+O\left(\frac{1}{(\log T)^{A}}\right) .
$$


To deduce Theorem 1.1 we use Beurling-Selberg functions (see Section 6 below) to relate the distribution function $\mathbb{P}_{T}(\log \zeta(\sigma+i t) \in \mathcal{R})$ to the characteristic function $\Phi_{\sigma, T}(u, v)$. We should note that any improvement in the range of validity of Theorem 2.1 would lead to an improved bound for the discrepancy $D_{\sigma}(T)$. Indeed, we can show that $D_{\sigma}(T) \ll 1 / L$ if the asymptotic formula (2.1) holds in the range $|u|,|v| \ll L$.

In order to investigate the distribution of large values of $\log |\zeta(\sigma+i t)|(\operatorname{or} \arg \zeta(\sigma+i t))$ and prove Theorem 1.2 , we study large complex moments of $\zeta(\sigma+i t)$ and compare them to the corresponding complex moments of $\zeta(\sigma, X)$. Define

$$
M_{z}(T):=\frac{1}{T} \int_{T}^{2 T}|\zeta(\sigma+i t)|^{z} d t
$$

Assuming the Riemann hypothesis, the first author [9] established an asymptotic formula for $M_{z}(T)$ uniformly in the range $|z| \ll(\log T)^{2 \sigma-1}$, and conjectured that such an asymptotic should hold in the extended range $|z| \ll(\log T)^{\sigma}$. The assumption of the Riemann hypothesis is necessary in this case, since $|\zeta(\sigma+i t)|^{z}$ is very large when $\sigma+i t$ is close to a zero of $\zeta(s)$ and $z$ is a negative real number. Also note that, when $\operatorname{Re}(z)$ is large, the moment $M_{z}(T)$ is heavily affected by the contribution of the points $t$ where $|\zeta(\sigma+i t)|$ is large. Thus, short of proving strong bounds for $|\zeta(\sigma+i t)|$ and without assuming the Riemann hypothesis, we cannot hope for asymptotics of the moments $M_{z}(T)$, except in a narrow range of values for $z$. To overcome this difficulty, we compute instead complex moments of $\zeta(\sigma+i t)$ after first removing a small set of "bad" points $t$ in $[T, 2 T]$, namely those close to zeros of $\zeta(s)$ and those for which $|\zeta(\sigma+i t)|$ is large. Using this method we obtain an asymptotic formula for these moments in the full conjectured range $|z| \ll(\log T)^{\sigma}$.

Theorem 2.2. Let $\frac{1}{2}<\sigma<1$ and $A \geq 1$ be fixed. There exist positive constants $b_{3}=b_{3}(\sigma, A)$ and $b_{4}=b_{4}(\sigma, A)$ and a set $\mathcal{E}(T) \subset[T, 2 T]$ of measure meas $(\mathcal{E}(T)) \leq$ $T \exp \left(-b_{3} \log T / \log \log T\right)$, such that for all complex numbers z with $|z| \leq b_{4}(\log T)^{\sigma}$ we have

$$
\frac{1}{T} \int_{[T, 2 T] \backslash \mathcal{E}(T)}|\zeta(\sigma+i t)|^{z} d t=\mathbb{E}\left(|\zeta(\sigma, X)|^{z}\right)+O\left(\frac{\mathbb{E}\left(|\zeta(\sigma, X)|^{\operatorname{Re}(z)}\right)}{(\log T)^{A}}\right),
$$

Moreover, the same asymptotic formula holds when $|\zeta(\sigma+i t)|^{z}$ and $|\zeta(\sigma, X)|^{z}$ are replaced by $\exp (z(\arg \zeta(\sigma+i t)))$ and $\exp (z(\arg \zeta(\sigma, X)))$ respectively.

When computing complex moments of $\zeta(\sigma+i t)$ the first step is to use the classical zero density estimates to approximate $\log \zeta(\sigma+i t)$ by a short Dirichlet polynomial for all $t \in[T, 2 T]$ except for a set of small measure (see Lemma 3.1 below). Let

$$
R_{Y}(\sigma+i t):=\sum_{p^{n} \leq Y} \frac{1}{n p^{n(\sigma+i t)}} \quad \text { and } \quad R_{Y}(\sigma, X):=\sum_{p^{n} \leq Y} \frac{X(p)^{n}}{n p^{\sigma n}} .
$$

We extract Theorems 2.1 and 2.2 from the following key proposition.

Proposition 2.3. Let $\frac{1}{2}<\sigma<1$ and $A \geq 1$ be fixed. Let $Y=(\log T)^{A}$. There exist positive constants $b_{5}=b_{5}(\sigma, A)>0$ and $b_{6}=b_{6}(\sigma, A)$ such that for all complex numbers 
$z_{1}, z_{2}$ with $\left|z_{1}\right|,\left|z_{2}\right| \leq b_{5}(\log T)^{\sigma}$ we have

$$
\begin{aligned}
\frac{1}{T} \int_{\mathcal{A}(T)} \exp \left(z_{1} R_{Y}(\sigma+i t)+z_{2} \overline{R_{Y}(\sigma+i t)}\right) d t \\
\quad=\mathbb{E}\left(\exp \left(z_{1} R_{Y}(\sigma, X)+z_{2} \overline{R_{Y}(\sigma, X)}\right)\right)+O\left(\exp \left(-b_{6} \frac{\log T}{\log \log T}\right)\right),
\end{aligned}
$$

where $\mathcal{A}(T)$ is the set of those $t \in[T, 2 T]$ such that $\left|R_{Y}(\sigma+i t)\right| \leq(\log T)^{1-\sigma} / \log \log T$.

Compared to earlier treatments our main innovation consists in the introduction of the condition $\left|R_{Y}(\sigma+i t)\right| \leq(\log T)^{1-\sigma} / \log \log T$ in $\mathcal{A}(T)$. Without this constraint the range of $\left|z_{1}\right|$ and $\left|z_{2}\right|$ in Proposition 2.3 would be reduced to $(\log T)^{2 \sigma-1}$.

Using Littlewood's Lemma (see equation (8.2) below), one can count the number of $a$-points of $\zeta(s)$ in the strip $\frac{1}{2}<\sigma_{1}<\sigma<\sigma_{2}<1, T \leq t \leq 2 T$, if one can estimate the integral

$$
\int_{T}^{2 T} \log |\zeta(\sigma+i t)-a| d t
$$

In [3], Borchsenius and Jessen proved the following asymptotic formula for this integral from which they deduced their result (1.2)

$$
\frac{1}{T} \int_{T}^{2 T} \log |\zeta(\sigma+i t)-a| d t \sim \mathbb{E}[\log |\zeta(\sigma, X)-a|], \text { as } T \rightarrow \infty .
$$

We improve on this result, obtaining the first effective error term for the integral (2.2).

Theorem 2.4. Let $\frac{1}{2}<\sigma<1$ and $a \neq 0$ be a complex number. Then,

$$
\frac{1}{T} \int_{T}^{2 T} \log |\zeta(\sigma+i t)-a| d t=\mathbb{E}[\log |\zeta(\sigma, X)-a|]+O\left(\frac{(\log \log T)^{2}}{(\log T)^{\sigma}}\right) .
$$

We should note that apart from the factor $(\log \log T)^{2}$, the error term in Theorem 2.4 is optimal in view of our bound for the discrepancy $D_{\sigma}(T)$ in Theorem 1.1 .

There are two main ingredients in the proof of Theorem 2.4. First, we use our result on $D_{\sigma}(T)$ to capture the main term. Secondly, to control the error term we need a completely uniform (but not necessarily very good) bound for the measure of those $t$ for which $\zeta(\sigma+i t)$ is very close to $a$. We achieve such an estimate by using the following $L^{2 k}$ bound.

Proposition 2.5. Let $\frac{1}{2}<\sigma \leq 2$ be fixed. Let $a \in \mathbb{C}$. There exists an absolute constant $C>0$ such that for every real number $k>0$ we have

$$
\frac{1}{T} \int_{T}^{2 T}|\log | \zeta(\sigma+i t)-\left.a\right|^{2 k} d t \ll(C k)^{4 k} .
$$

In order to study $a$-points to the left of the half-line, Selberg obtains a similar proposition when $\sigma=\frac{1}{2}$. His argument depends on the rapid rate of change of the phase of $\zeta(\sigma+i t)$ when $\sigma \leq \frac{1}{2}$ (for $\sigma<\frac{1}{2}$ this follows from the Riemann hypothesis) and does not generalize to any line with $\sigma>\frac{1}{2}$ (see [17], Chapter 8, in particular the discussion on page 119). Our treatment depends on a careful use of Jensen's formula. Proposition 2.5 
bears some resemblance to a result obtained by Guo to study zeros of $\zeta^{\prime}(s)$. Our result is more refined, in particular our treatment removes the loss of a power of $\log \log T$.

\section{Preliminary Lemmas}

In this section we collect together several preliminary results that will be useful in our subsequent work.

Lemma 3.1 (Lemma 2.2 of [4]). Let $\frac{1}{2}<\sigma \leq 1$ be fixed and $3 \leq Y \leq T / 2$. For $t \in[T, 2 T]$ except outside a set of measure $\ll T^{5 / 4-\sigma / 2} Y \log ^{5} T$ we have

$$
\log \zeta(\sigma+i t)=R_{Y}(\sigma+i t)+O\left(Y^{-(\sigma-1 / 2) / 2} \log ^{3} T\right) .
$$

Lemma 3.2. Let $2 \leq y \leq z$. For any positive integer $k$ that $i s \leq \log T /(3 \log z)$ we have

$$
\frac{1}{T} \int_{T}^{2 T}\left|\sum_{y \leq p \leq z} \frac{1}{p^{\sigma+i t}}\right|^{2 k} d t \ll k !\left(\sum_{y \leq p \leq z} \frac{1}{p^{2 \sigma}}\right)^{k}+T^{-1 / 3}
$$

Additionally, for any positive integer $k$ we have

$$
\mathbb{E}\left(\left|\sum_{y \leq p \leq z} \frac{X(p)}{p^{\sigma}}\right|^{2 k}\right) \ll k !\left(\sum_{y \leq p \leq z} \frac{1}{p^{2 \sigma}}\right)^{k} .
$$

Proof. The first assertion of the lemma is Lemma 4.2 of [9]. Next, note that

$$
\mathbb{E}\left(\left|\sum_{y \leq p \leq z} \frac{X(p)}{p^{\sigma}}\right|^{2 k}\right)=\sum_{\substack{y \leq p_{1}, \ldots, p_{k} \leq z \\ y \leq q_{1}, \ldots, q_{k} \leq z}} \frac{1}{\left(p_{1} \cdots p_{k} q_{1} \cdots q_{k}\right)^{\sigma}} \mathbb{E}\left(X\left(p_{1}\right) \cdots X\left(p_{k}\right) \overline{X\left(q_{1}\right) \cdots X\left(q_{k}\right)}\right) .
$$

Since the $X(p)$ 's are independent random variables uniformly distributed on the unit circle the only terms that contribute to the above sum are those where $p_{1} \cdots p_{k}=$ $q_{1} \cdots q_{k}$. The contribution from these terms is

$$
\ll k !\left(\sum_{y \leq p \leq z} \frac{1}{p^{2 \sigma}}\right)^{k} .
$$

Lemma 3.3. Let $\frac{1}{2}<\sigma<1$ and $A \geq 1$ be fixed. Also, let $Y=(\log T)^{A}$ and $k$ be an integer that satisfies $2 \leq k \leq \log T /(6 A \log \log T)$. Then there exists a constant $a(\sigma)>0$ such that

$$
\frac{1}{T} \int_{T}^{2 T}\left|R_{Y}(\sigma+i t)\right|^{2 k} d t \ll\left(\frac{a(\sigma) k^{1-\sigma}}{(\log k)^{\sigma}}\right)^{2 k} .
$$

Additionally, for any integer $k \geq 2$ we have

$$
\mathbb{E}\left(\left|R_{Y}(\sigma, X)\right|^{2 k}\right) \ll\left(\frac{a(\sigma) k^{1-\sigma}}{(\log k)^{\sigma}}\right)^{2 k} .
$$


Proof. We will only prove the first assertion; the second follows from a similar argument. Plainly,

$$
\begin{gathered}
\int_{T}^{2 T}\left|R_{Y}(\sigma+i t)\right|^{2 k} d t \leq 9^{k}\left(\int_{T}^{2 T}\left|\sum_{p \leq k \log k} \frac{1}{p^{\sigma+i t}}\right|^{2 k} d t+\int_{T}^{2 T}\left|\sum_{k \log k \leq p \leq Y} \frac{1}{p^{\sigma+i t}}\right|^{2 k} d t\right. \\
\left.+O\left(T \log ^{2 k} \zeta(2 \sigma)\right)\right) .
\end{gathered}
$$

By Lemma 3.2 and the prime number theorem we have

$$
\frac{1}{T} \int_{T}^{2 T}\left|\sum_{k \log k \leq p \leq Y} \frac{1}{p^{\sigma+i t}}\right|^{2 k} d t \ll k !\left(\sum_{k \log k \leq p \leq Y} \frac{1}{p^{2 \sigma}}\right)^{k}+T^{-1 / 3} \ll k^{k}\left(\frac{(k \log 2 k)^{1-2 \sigma}}{(2 \sigma-1) \log k}\right)^{k} .
$$

Next, note that for fixed $1 / 2<\sigma<1$

$$
\frac{1}{T} \int_{T}^{2 T}\left|\sum_{p \leq k \log k} \frac{1}{p^{\sigma+i t}}\right|^{2 k} d t \leq\left(\sum_{p \leq k \log 2 k} \frac{1}{p^{\sigma}}\right)^{2 k} \ll\left(\frac{(k \log k)^{1-\sigma}}{(1-\sigma) \log k}\right)^{2 k},
$$

by the prime number theorem. Inserting the two estimates above into (3.2) completes the proof.

Lemma 3.4. Let $\frac{1}{2}<\sigma<1$ and $A \geq 1$ be fixed, and let $Y=(\log T)^{A}$. Then there exists a constant $B=B(\sigma, A)$ such that

$$
\mathbb{P}_{T}\left(\left|R_{Y}(\sigma+i t)\right| \geq \frac{(\log T)^{1-\sigma}}{\log \log T}\right) \ll \exp \left(-B \frac{\log T}{\log \log T}\right)
$$

and

$$
\mathbb{P}\left(\left|R_{Y}(\sigma, X)\right| \geq \frac{(\log T)^{1-\sigma}}{\log \log T}\right) \ll \exp \left(-B \frac{\log T}{\log \log T}\right) .
$$

Proof. We will only prove the first assertion; the second follows from a similar argument. Let $2 \leq k \leq \log T /(6 A \log \log T)$ be an integer. Then, Lemma 3.3 implies that

$$
\begin{aligned}
\mathbb{P}_{T}\left(\left|R_{Y}(\sigma+i t)\right| \geq \frac{(\log T)^{1-\sigma}}{\log \log T}\right) & \leq\left(\frac{(\log T)^{1-\sigma}}{\log \log T}\right)^{-2 k} \frac{1}{T} \int_{T}^{2 T}\left|R_{Y}(\sigma+i t)\right|^{2 k} d t \\
& \ll\left(\frac{C k^{1-\sigma} \log \log T}{(\log k)^{\sigma}(\log T)^{1-\sigma}}\right)^{2 k} .
\end{aligned}
$$

Choosing $k=\left[\log T /\left(C_{1} \log \log T\right)\right]$, where $C_{1}=6 A(1+C)^{1 /(1-\sigma)}$, yields the desired bound.

Lemma 3.5. Let $\frac{1}{2}<\sigma \leq 1$ and $A \geq 1$ be fixed, and let $Y=(\log T)^{A}$. Then, for any positive integers $k, \ell$ such that $k+\ell \leq(\log T) /(6 A \log \log T)$, we have

$$
\begin{aligned}
\frac{1}{T} \int_{T}^{2 T}\left(R_{Y}(\sigma+i t)\right)^{k} & \cdot\left(\overline{R_{Y}(\sigma+i t)}\right)^{\ell} d t \\
& =\mathbb{E}\left(\left(R_{Y}(\sigma, X)\right)^{k} \cdot\left(\overline{R_{Y}(\sigma, X)}\right)^{\ell}\right)+O\left(\frac{Y^{k+\ell}}{\sqrt{T}}\right) .
\end{aligned}
$$


DISCREPANCY BOUNDS FOR THE DISTRIBUTION OF THE RIEMANN ZETA-FUNCTION 9

Proof. See Lemma 3.4 in Tsang's thesis [17].

4. Complex moments of $\zeta(\sigma+i t)$ : Proofs of Theorems 2.1 and 2.2

We begin by proving Proposition 2.3 .

Proof of Proposition 2.3. Let $k=\max \left\{\left|z_{1}\right|,\left|z_{2}\right|\right\}$, and $N=[\log T /(D(\log \log T))]$ where $D$ is a suitably large constant. Then, we have

$$
\begin{aligned}
& \frac{1}{T} \int_{\mathcal{A}(T)} \exp \left(z_{1} R_{Y}(\sigma+i t)+z_{2} \overline{R_{Y}(\sigma+i t)}\right) d t \\
& =\sum_{j+\ell \leq N} \frac{z_{1}^{j} z_{2}^{\ell}}{j ! \ell !} \frac{1}{T} \int_{\mathcal{A}(T)}\left(R_{Y}(\sigma+i t)\right)^{j}\left(\overline{R_{Y}(\sigma+i t)}\right)^{\ell} d t+E_{1}
\end{aligned}
$$

where

$$
\begin{aligned}
E_{1} & \ll \sum_{j+\ell \geq N} \frac{k^{j+\ell}}{j ! \ell !} \frac{1}{T} \int_{\mathcal{A}(T)}\left|R_{Y}(\sigma+i t)\right|^{j+\ell} \mathrm{d} t \leq \sum_{n \geq N} \frac{k^{n}}{n !}\left(\frac{(\log T)^{1-\sigma}}{\log \log T}\right)^{n} \sum_{j \leq n} \frac{n !}{j !(n-j) !} \\
& \leq \sum_{n \geq N} \frac{1}{n !}\left(\frac{2 k(\log T)^{1-\sigma}}{\log \log T}\right)^{n} \leq \sum_{n \geq N}\left(\frac{6 k(\log T)^{1-\sigma}}{N \log \log T}\right)^{n} \ll e^{-N},
\end{aligned}
$$

using Stirling's formula along with the fact that $\left|R_{Y}(\sigma+i t)\right| \leq(\log T)^{1-\sigma} / \log \log T$ for $t \in \mathcal{A}(T)$.

Let $\mathcal{S}(T)=\{T \leq t \leq 2 T: t \notin \mathcal{A}(T)\}$. If $j+\ell \leq N$ then using Lemmas 3.3 and 3.4 along with the Cauchy-Schwarz inequality we get

$$
\begin{aligned}
& \frac{1}{T} \int_{\mathcal{S}(T)}\left(R_{Y}(\sigma+i t)\right)^{j}\left(\overline{R_{Y}(\sigma+i t)}\right)^{\ell} d t \\
& \leq\left(\frac{\operatorname{meas}(\mathcal{S}(T))}{T}\right)^{1 / 2}\left(\frac{1}{T} \int_{T}^{2 T}\left|R_{Y}(\sigma+i t)\right|^{2(j+\ell)} d t\right)^{1 / 2} \\
& \ll \exp \left(-B \frac{\log T}{2 \log \log T}\right)\left(C \frac{(j+\ell)^{1-\sigma}}{(\log (j+\ell+2))^{\sigma}}\right)^{j+\ell},
\end{aligned}
$$

for some positive constants $B=B(\sigma, A)$ and $C=C(\sigma)$. Inserting this bound in equation (4.1) we deduce

$$
\begin{aligned}
& \frac{1}{T} \int_{\mathcal{A}(T)} \exp \left(z_{1} R_{Y}(\sigma+i t)+z_{2} \overline{R_{Y}(\sigma+i t)}\right) d t \\
& =\sum_{j+\ell \leq N} \frac{z_{1}^{j} z_{2}^{\ell}}{j ! \ell !} \frac{1}{T} \int_{T}^{2 T}\left(R_{Y}(\sigma+i t)\right)^{j}\left(\overline{R_{Y}(\sigma+i t)}\right)^{\ell} d t+E_{2},
\end{aligned}
$$


where

$$
\begin{aligned}
E_{2} & \ll \exp \left(-B \frac{\log T}{2 \log \log T}\right) \sum_{j+\ell \leq N} \frac{k^{j+\ell}}{j ! \ell !}\left(C \frac{(j+\ell)^{1-\sigma}}{(\log (j+\ell+2))^{\sigma}}\right)^{j+\ell}+e^{-N} \\
& \ll \exp \left(-B \frac{\log T}{2 \log \log T}\right) \sum_{n \leq N} \frac{1}{n !}\left(2 C \frac{k N^{1-\sigma}}{(\log (N+2))^{\sigma}}\right)^{n}+e^{-N} \\
& \ll \exp \left(-B \frac{\log T}{2 \log \log T}\right) \exp \left(2 C \frac{k N^{1-\sigma}}{(\log (N+2))^{\sigma}}\right)+e^{-N} \\
& \ll \exp \left(-B \frac{\log T}{4 \log \log T}\right)+e^{-N},
\end{aligned}
$$

if $D$ is suitably large and $k \leq c_{0}(\log T)^{\sigma}$ where $c_{0}$ is suitably small.

Now, for all $j+\ell \leq N$, we have by Lemma 3.5 that

$$
\begin{aligned}
\frac{1}{T} \int_{T}^{2 T}\left(R_{Y}(\sigma+i t)\right)^{j}\left(\overline{R_{Y}(\sigma+i t)}\right)^{\ell} d t= & \mathbb{E}\left(\left(R_{Y}(\sigma, X)\right)^{j}\left(\overline{R_{Y}(\sigma, X)}\right)^{\ell}\right) \\
& +O\left(\frac{Y^{j+\ell}}{\sqrt{T}}\right) .
\end{aligned}
$$

Note that $Y^{2 N} \ll T^{1 / 4}$ if $D$ is suitably large. By this, (4.2), and (4.3) we obtain

$$
\begin{aligned}
& \frac{1}{T} \int_{\mathcal{A}(T)} \exp \left(z_{1} R_{Y}(\sigma+i t)+z_{2} \overline{R_{Y}(\sigma+i t)}\right) d t \\
& =\sum_{j+\ell \leq N} \frac{z_{1}^{j} z_{2}^{\ell}}{j ! \ell !} \mathbb{E}\left(\left(R_{Y}(\sigma, X)\right)^{j}\left(\overline{R_{Y}(\sigma, X)}\right)^{\ell}\right)+O\left(\exp \left(-\frac{B}{4} \frac{\log T}{\log \log T}\right)\right) .
\end{aligned}
$$

Furthermore, by Lemma 3.3 ,

$$
\mathbb{E}\left(\left|R_{Y}(\sigma, X)\right|^{k}\right) \ll\left(C \frac{k^{1-\sigma}}{(\log k)^{\sigma}}\right)^{k},
$$

for all $k \geq 2$. Therefore, the main term on the right-hand side of (4.4) equals

$$
\mathbb{E}\left(\exp \left(z_{1} R_{Y}(\sigma, X)+z_{2} \overline{R_{Y}(\sigma, X)}\right)\right)+E_{3}
$$

where

$$
E_{3} \ll \sum_{n \geq N} \frac{1}{n !}\left(\frac{2 C k n^{1-\sigma}}{(\log n)^{\sigma}}\right)^{n} \leq \sum_{n \geq N}\left(\frac{6 C k}{(n \log n)^{\sigma}}\right)^{n} \leq \sum_{n \geq N}\left(\frac{6 C k}{(N \log N)^{\sigma}}\right)^{n} \ll e^{-N} .
$$

This completes the proof.

Before proving Theorems 2.1 and 2.2, we need the following lemma which shows that the characteristic function of the random variable $\log \zeta(\sigma, X)$ is well approximated by that of $R_{Y}(\sigma, X)$ in a certain range that depends on $Y$. 
DISCREPANCY BOUNDS FOR THE DISTRIBUTION OF THE RIEMANN ZETA-FUNCTION 11

Lemma 4.1. Let $Y$ be a large positive real number, and $s$ be a complex number such that $|s| \leq Y^{\sigma-1 / 2}$. Then we have

$$
\mathbb{E}\left(|\zeta(\sigma, X)|^{s}\right)=\mathbb{E}\left(\exp \left(s \operatorname{Re}\left(R_{Y}(\sigma, X)\right)\right)\right)+O\left(\mathbb{E}\left(|\zeta(\sigma, X)|^{\operatorname{Re}(s)}\right) \frac{|s|}{Y^{\sigma-1 / 2}}\right) .
$$

Moreover, if $u, v$ are real numbers such that $|u|+|v| \leq Y^{\sigma-1 / 2}$, then

$$
\Phi_{\sigma}^{\mathrm{rand}}(u, v)=\mathbb{E}\left(\exp \left(i u \operatorname{Re} R_{Y}(\sigma, X)+i v \operatorname{Im} R_{Y}(\sigma, X)\right)\right)+O\left(\frac{|u|+|v|}{Y^{\sigma-1 / 2}}\right) .
$$

Proof. Let $z$ be a complex number with $|z| \leq Y^{\sigma-1 / 2}$. Using that

$$
\sum_{\substack{n \geq 2 \\ p^{n}>Y}} \frac{1}{p^{\sigma n}} \ll \frac{1}{Y^{\sigma-1 / 2}}
$$

we obtain

$\mathbb{E}(\exp (z \operatorname{Re} \log \zeta(\sigma, X)))=\mathbb{E}\left(\exp \left(z \operatorname{Re}\left(R_{Y}(\sigma, X)\right)+z \operatorname{Re} \sum_{p>Y} \frac{X(p)}{p^{\sigma}}+O\left(\frac{|z|}{Y^{\sigma-1 / 2}}\right)\right)\right)$.

Furthermore, if $p>Y$ then $|z|<p^{\sigma}$ and hence

$$
\mathbb{E}\left(\exp \left(z \operatorname{Re} \frac{X(p)}{p^{\sigma}}\right)\right)=\mathbb{E}\left(1+z \operatorname{Re} \frac{X(p)}{p^{\sigma}}+O\left(\frac{|z|^{2}}{p^{2 \sigma}}\right)\right)=1+O\left(\frac{|z|^{2}}{p^{2 \sigma}}\right) .
$$

The independence of the $X(p)$ 's together with the fact that $\sum_{p>Y} p^{-2 \sigma} \ll Y^{1-2 \sigma}$ imply that

$$
\mathbb{E}(\exp (z \operatorname{Re} \log \zeta(\sigma, X)))=\mathbb{E}\left(\exp \left(z \operatorname{Re}\left(R_{Y}(\sigma, X)\right)+O\left(\frac{|z|}{Y^{\sigma-1 / 2}}\right)\right)\right),
$$

from which (4.5) follows. To obtain (4.6) one also uses that

$$
\mathbb{E}(\exp (z \operatorname{Im} \log \zeta(\sigma, X)))=\mathbb{E}\left(\exp \left(z \operatorname{Im}\left(R_{Y}(\sigma, X)\right)+O\left(\frac{|z|}{Y^{\sigma-1 / 2}}\right)\right)\right),
$$

which can be obtained along similar lines.

Proof of Theorem 2.1. Let $Y=(\log T)^{B /(\sigma-1 / 2)}$ where $B=B(A)$ is a suitably large constant that will be chosen later. Then it follows from Lemma 3.1 that

$$
\log \zeta(\sigma+i t)=R_{Y}(\sigma+i t)+O\left(\frac{1}{(\log T)^{B / 2-3}}\right)
$$

for all $t \in[T, 2 T]$ except a set of measure $T^{1-d(\sigma)}$ for some constant $d(\sigma)>0$. Let $\mathcal{B}(T)$ be the set of $t \in[T, 2 T]$ such that (4.8) holds. Note that $\left|e^{i b}-e^{i a}\right|=\left|\int_{a}^{b} e^{i x} d x\right| \leq|b-a|$. 
Therefore, we obtain

$$
\begin{aligned}
\Phi_{\sigma, T}(u, v) & =\frac{1}{T} \int_{\mathcal{B}(T)} \exp (i u \operatorname{Re} \log \zeta(\sigma+i t)+i v \operatorname{Im} \log \zeta(\sigma+i t)) d t+O\left(T^{-d(\sigma)}\right) \\
& =\frac{1}{T} \int_{\mathcal{B}(T)} \exp \left(i u \operatorname{Re} R_{Y}(\sigma+i t)+i v \operatorname{Im} R_{Y}(\sigma+i t)\right) d t+O\left(\frac{1}{(\log T)^{B / 2-4}}\right) \\
& =\frac{1}{T} \int_{T}^{2 T} \exp \left(i u \operatorname{Re} R_{Y}(\sigma+i t)+i v \operatorname{Im} R_{Y}(\sigma+i t)\right) d t+O\left(\frac{1}{(\log T)^{B / 2-4}}\right) .
\end{aligned}
$$

Let $\mathcal{A}(T)$ be as in Proposition 2.3. Then, by Lemma 3.4 and Proposition 2.3, taking $z_{1}=\frac{i}{2}(u-i v)$ and $z_{2}=\frac{i}{2}(u+i v)$ there, we get

$$
\begin{aligned}
& \frac{1}{T} \int_{T}^{2 T} \exp \left(i u \operatorname{Re} R_{Y}(\sigma+i t)+i v \operatorname{Im} R_{Y}(\sigma+i t)\right) d t \\
& =\frac{1}{T} \int_{\mathcal{A}(T)} \exp \left(i u \operatorname{Re} R_{Y}(\sigma+i t)+i v \operatorname{Im} R_{Y}(\sigma+i t)\right) d t+O\left(\frac{1}{(\log T)^{B}}\right) \\
& =\mathbb{E}\left(\exp \left(i u \operatorname{Re} R_{Y}(\sigma, X)+i v \operatorname{Im} R_{Y}(\sigma, X)\right)\right)+O\left(\frac{1}{(\log T)^{B}}\right) .
\end{aligned}
$$

Finally, using (4.6) we deduce

$$
\mathbb{E}\left(\exp \left(i u \operatorname{Re} R_{Y}(\sigma, X)+i v \operatorname{Im} R_{Y}(\sigma, X)\right)\right)=\Phi_{\sigma}^{\mathrm{rand}}(u, v)+O\left(\frac{1}{(\log T)^{B-1}}\right) .
$$

Choosing $B=2 A+8$, and collecting the above estimates completes the proof.

Proof of Theorem 2.2. As in the proof of Theorem 2.1, let $Y=(\log T)^{B /(\sigma-1 / 2)}$ where $B=2 A+8$, and $\mathcal{B}(T)$ be the set of $t \in[T, 2 T]$ such that (4.8) holds. Then, by Lemma 3.1 meas $([T, 2 T] \backslash \mathcal{B}(T)) \ll T^{1-d(\sigma)}$ for some constant $d(\sigma)>0$. Moreover, let $\mathcal{A}(T)$ be as in Proposition 2.3. We define

$$
\mathcal{E}(T):=[T, 2 T] \backslash(\mathcal{A}(T) \cap \mathcal{B}(T)) .
$$

Then, it follows from Lemma 3.4 that

$$
\operatorname{meas}(\mathcal{E}(T)) \ll T \exp \left(-b_{0} \frac{\log T}{\log \log T}\right),
$$

for some positive constant $b_{0}=b_{0}(\sigma, A)$.

Now, by (4.8) we get

$$
\begin{aligned}
\frac{1}{T} \int_{[T, 2 T] \backslash \mathcal{E}(T)}|\zeta(\sigma+i t)|^{z} d t & =\frac{1}{T} \int_{\mathcal{A}(T) \cap \mathcal{B}(T)} \exp \left(z \operatorname{Re}\left(R_{Y}(\sigma+i t)\right)+O\left(\frac{1}{(\log T)^{A}}\right)\right) d t \\
& =\frac{1}{T} \int_{\mathcal{A}(T) \cap \mathcal{B}(T)} \exp \left(z \operatorname{Re}\left(R_{Y}(\sigma+i t)\right)\right) d t+E_{4}
\end{aligned}
$$


where

$$
\begin{aligned}
E_{4} & \ll \frac{1}{T(\log T)^{A}} \int_{\mathcal{A}(T)} \exp \left(\operatorname{Re}(z) \operatorname{Re}\left(R_{Y}(\sigma+i t)\right)\right) d t \\
& \ll \frac{1}{(\log T)^{A}} \mathbb{E}\left(\exp \left(\operatorname{Re}(z) \operatorname{Re}\left(R_{Y}(\sigma, X)\right)\right)\right) \\
& \ll \frac{1}{(\log T)^{A}} \mathbb{E}\left(|\zeta(\sigma, X)|^{\operatorname{Re}(z)}\right),
\end{aligned}
$$

by Proposition 2.3 and Lemma 4.1

On the other hand, since meas $([T, 2 T] \backslash \mathcal{B}(T)) \ll T^{1-d(\sigma)}$, and $\left|R_{Y}(\sigma+i t)\right| \leq$ $(\log T)^{1-\sigma} / \log \log T$ for all $t \in \mathcal{A}(T)$ we deduce that

$$
\begin{aligned}
\frac{1}{T}\left(\int_{\mathcal{A}(T)}-\int_{\mathcal{A}(T) \cap \mathcal{B}(T)}\right) \exp \left(z \operatorname{Re}\left(R_{Y}(\sigma+i t)\right)\right) d t & \ll T^{-d(\sigma)} \exp \left(\operatorname{Re}(z) \frac{(\log T)^{1-\sigma}}{\log \log T}\right) \\
& \ll T^{-d(\sigma) / 2} .
\end{aligned}
$$

Furthermore, combining Proposition 2.3 and Lemma 4.1 we obtain

$$
\begin{aligned}
\frac{1}{T} \int_{\mathcal{A}(T)} \exp \left(z \operatorname{Re}\left(R_{Y}(\sigma+i t)\right)\right) d t & =\mathbb{E}\left(\exp \left(z \operatorname{Re}\left(R_{Y}(\sigma, X)\right)\right)\right)+O\left(\frac{1}{(\log T)^{A}}\right) \\
& =\mathbb{E}\left(|\zeta(\sigma, X)|^{z}\right)+O\left(\frac{1}{(\log T)^{A}} \mathbb{E}\left(|\zeta(\sigma, X)|^{\operatorname{Re}(z)}\right)\right)
\end{aligned}
$$

The result follows upon inserting the estimates (4.11), (4.12) and (4.13) in (4.10).

\section{5. $L^{2 k}$ NORM of $\log \zeta(\sigma+i t)-a$ : Proof of Proposition 2.5}

As a special case of Lemma 2.2.1 of Guo [5], which itself is a generalization of a lemma of Landau (see [10] or Lemma $\alpha$ from Chapter III of [16]), we have

Lemma 5.1. Let $0<r \ll 1$. Also, let $s_{0}=\sigma_{0}+i t$ and suppose $f(z)$ is analytic in $\left|z-s_{0}\right| \leq r$. Define

$$
M_{r}\left(s_{0}\right)=\max _{\left|z-s_{0}\right| \leq r}\left|\frac{f(z)}{f\left(s_{0}\right)}\right|+3 \quad \text { and } \quad N_{r}\left(s_{0}\right)=\sum_{\left|\varrho-s_{0}\right| \leq r} 1,
$$

where the last sum runs over the zeros, $\varrho$, of $f(z)$ in the closed disk of radius $r$ centered at $s_{0}$. Then for $0<\delta<r / 2$ and $\left|z-s_{0}\right| \leq r-2 \delta$ we have

$$
\frac{f^{\prime}}{f}(z)=\sum_{\left|\rho-s_{0}\right| \leq r-\delta} \frac{1}{s-\rho}+O\left(\frac{1}{\delta^{2}}\left(\log M_{r}\left(s_{0}\right)+N_{r-\delta}\left(s_{0}\right)(\log 1 / \delta+1)\right)\right) .
$$

In the following we take

$$
f(z)=f_{a}(z)=\left\{\begin{array}{l}
(\zeta(z)-a) /(1-a) \text { if } a \neq 1 \\
2^{z}(\zeta(z)-1) \text { if } a=1
\end{array}\right.
$$


We also choose

$$
\delta=(\sigma-1 / 2) / 5 \quad \text { and } \quad r=\sigma_{0}-(\sigma+1 / 2) / 2,
$$

where $\sigma_{0}$ is taken to be large enough (depending on $a$ ) so that $\left|f\left(\sigma_{0}+i t\right)\right| \geq 1 / 10$ and $\min _{\rho_{a}}\left|s_{0}-\rho_{a}\right| \geq 1 / 10$ uniformly in $t$.

For $\left|z-s_{0}\right| \leq r-2 \delta$ Lemma 5.1 yields

$$
\frac{\zeta^{\prime}(z)}{\zeta(z)-a}=\sum_{\left|\rho_{a}-s_{0}\right| \leq r-\delta} \frac{1}{z-\rho_{a}}+O\left(\frac{1}{\delta^{2}}\left(\log M_{r}\left(s_{0}\right)+N_{r-\delta}\left(s_{0}\right)\right)(\log 1 / \delta+1)\right) .
$$

Lemma 5.2. Let $1 / 2<\sigma \leq 2$ be fixed. Also, let $\delta, r$, and $\sigma_{0}$ be as in (5.2). For $t$ sufficiently large we have

$$
\log |\zeta(\sigma+i t)-a|=\sum_{\left|\rho_{a}-s_{0}\right| \leq r-\delta} \log \left|\sigma+i t-\rho_{a}\right|+O\left(\log M_{r}\left(s_{0}\right)\right) .
$$

Proof. Let $f(z)$ be as in (5.1). First, note that Jensen's formula gives

$$
\int_{0}^{r} N_{x}\left(s_{0}\right) \frac{d x}{x}=\frac{1}{2 \pi} \int_{0}^{2 \pi} \log \left|f\left(r e^{i \theta}+s_{0}\right)\right| d \theta-\log \left|f\left(s_{0}\right)\right| .
$$

Observe that

$$
\int_{0}^{r} N_{x}\left(s_{0}\right) \frac{d x}{x} \geq \int_{r-\delta}^{r} N_{x}\left(s_{0}\right) \frac{d x}{x} \geq \frac{\delta}{r} N_{r-\delta}\left(s_{0}\right) .
$$

By this and the bound $\log \left|f\left(s_{0}\right)\right| \geq \log 1 / 10$ we have

$$
N_{r-\delta}\left(s_{0}\right) \leq \frac{r}{\delta}\left(\frac{1}{2 \pi} \int_{0}^{2 \pi} \log \left|f\left(r e^{i \theta}+s_{0}\right)\right| d \theta-\log \left|f\left(s_{0}\right)\right|\right) \leq \frac{r}{\delta}\left(\log M_{r}\left(s_{0}\right)+\log 10\right) .
$$

Applying this estimate in (5.3) and noting that $\delta \gg 1$ and $r \ll 1$ we have

$$
\frac{\zeta^{\prime}(z)}{\zeta(z)-a}=\sum_{\left|\rho_{a}-s_{0}\right| \leq r-\delta} \frac{1}{z-\rho_{a}}+O\left(\log M_{r}\left(s_{0}\right)\right)
$$

for $\left|z-s_{0}\right| \leq r-2 \delta$. In particular, this formula is valid along the line segment that connects $s$ to $s_{0}$. Hence, integrating the above equation from $s$ to $s_{0}$ and taking real parts gives

$\log |\zeta(s)-a|-\log \left|\zeta\left(s_{0}\right)-a\right|=\sum_{\left|\rho_{a}-s_{0}\right| \leq r-\delta}\left(\log \left|s-\rho_{a}\right|-\log \left|s_{0}-\rho_{a}\right|\right)+O\left(\log M_{r}\left(s_{0}\right)\right)$.

By the choice of $\sigma_{0}$ we have

$$
\log \left|\zeta\left(s_{0}\right)-a\right|=O(1) \quad \text { and } \quad \log \left|s_{0}-\rho_{a}\right|=O(1)
$$

Thus,

$$
\log |\zeta(s)-a|=\sum_{\left|\rho_{a}-s_{0}\right| \leq r-\delta} \log \left|s-\rho_{a}\right|+O\left(N_{r-\delta}\left(s_{0}\right)+\log M_{r}\left(s_{0}\right)\right) .
$$

Applying (5.4) to the error term completes the proof. 
Lemma 5.3. Let $\frac{1}{2}<\sigma \leq 2$ be fixed. Also, let $r, \delta$, and $\sigma_{0}$ be as in (5.2). Then there exists an absolute constant $C>0$ such that for any real number $k \geq \frac{1}{2}$ we have

$$
\frac{1}{T} \int_{T}^{2 T}\left(\sum_{\left|\rho_{a}-s_{0}\right| \leq r-\delta}|\log | \sigma+i t-\rho_{a}||\right)^{2 k} d t \ll \Gamma(2 k+1)\left(C \log M_{r+\delta}\left(s_{0}\right)\right)^{2 k} .
$$

Proof. Define $D_{R}(z)$ to be the closed disc of radius $R$ centered at $z$. For $n=\lfloor T\rfloor, \ldots,\lfloor 2 T\rfloor+$ 1 let

$$
\mathcal{D}_{n}=\bigcup_{\ell=0}^{\lfloor 1 / \sqrt{\delta}\rfloor+1} D_{r}\left(\sigma_{0}+i(n+\ell \cdot \sqrt{\delta})\right)
$$

Observe that

$D_{r-\delta}\left(\sigma_{0}+i n\right) \bigcup\left\{z: n \leq \operatorname{Im} z \leq n+\sqrt{\delta}, \sigma_{0}-(r-\delta) \leq \operatorname{Re} z \leq \sigma_{0}+r-\delta\right\} \subset D_{r}\left(\sigma_{0}+i n\right)$.

Next, note that

$\left\{z: n+\sqrt{\delta} \leq \operatorname{Im} z \leq n+2 \sqrt{\delta}, \sigma_{0}-(r-\delta) \leq \operatorname{Re} z \leq \sigma_{0}+r-\delta\right\} \subset D_{r}\left(\sigma_{0}+i(n+\sqrt{\delta})\right)$,

and so on. Hence, by construction

$$
\bigcup_{n \leq t \leq n+1} D_{r-\delta}\left(\sigma_{0}+i t\right) \subset \mathcal{D}_{n}
$$

This implies that

$$
\begin{aligned}
\int_{T}^{2 T}\left(\sum_{\left|\rho_{a}-s_{0}\right| \leq r-\delta}|\log | \sigma+i t-\rho_{a}||\right)^{2 k} d t & \leq \sum_{n=\lfloor T\rfloor}^{\lfloor 2 T\rfloor+1} \int_{n}^{n+1}\left(\sum_{\left|\rho_{a}-s_{0}\right| \leq r-\delta}|\log | \sigma+i t-\rho_{a}||\right)^{2 k} d t \\
& \leq \sum_{n=\lfloor T\rfloor}^{\lfloor 2 T\rfloor+1} \int_{n}^{n+1}\left(\sum_{\rho_{a} \in \mathcal{D}_{n}}|\log | \sigma+i t-\rho_{a}||\right)^{2 k} d t .
\end{aligned}
$$

Applying Minkowski's inequality to the right-hand side we get that

$$
\begin{aligned}
\int_{T}^{2 T}\left(\sum_{\left|\rho_{a}-s_{0}\right| \leq r-\delta}|\log | \sigma+i t\right. & \left.-\rho_{a}||\right)^{2 k} d t \\
& \leq \sum_{n=\lfloor T\rfloor}^{\lfloor 2 T\rfloor+1}\left(\sum_{\rho_{a} \in \mathcal{D}_{n}}\left(\int_{n}^{n+1}|\log | \sigma+i t-\rho_{a}||^{2 k} d t\right)^{1 /(2 k)}\right)^{2 k} .
\end{aligned}
$$

We now estimate the inner integral on the right-hand side. We have for $n \leq t \leq n+1$ and $\rho_{a} \in \mathcal{D}_{n}$ that

$$
\left|t-\gamma_{a}\right| \leq\left|\sigma+i t-\rho_{a}\right| \leq c
$$

for some absolute constant $c=c(a)>1$. So for $n \leq t \leq n+1$ and $\rho_{a} \in \mathcal{D}_{n}$ we get that

$$
|\log | \sigma+i t-\rho_{a}||^{2 k} \leq|\log | t-\gamma_{a}||^{2 k}+|\log c|^{2 k} \text {. }
$$


Also, for $\rho_{a} \in \mathcal{D}_{n}$ we have $n-r \leq \gamma_{a} \leq n+r+2$. Thus,

$$
\begin{aligned}
\int_{n}^{n+1}|\log | t-\gamma_{a}||^{2 k} d t & \leq \int_{n-r}^{n+r+2}|\log | t-\gamma_{a}||^{2 k} d t \\
& \leq 2 \int_{0}^{2 r+2}|\log x|^{2 k} d x \\
& =2 \Gamma(2 k+1)+O\left((\log (2 r+2))^{2 k}\right) .
\end{aligned}
$$

Next, note that the set $\mathcal{D}_{n}$ consists of $\ll 1 / \sqrt{\delta}=O(1)$ disks, each of radius $r$. Arguing as in (5.4), we see that each one contains $\ll \delta^{-1} \log M_{r+\delta}\left(s_{0}\right) \ll \log M_{r+\delta}\left(s_{0}\right)$ zeros. Hence, by this, (5.5), (5.6), and (5.7) we see that

$$
\begin{aligned}
& \int_{T}^{2 T}\left(\sum_{\left|\rho_{a}-s_{0}\right| \leq r-\delta}|\log | \sigma+i t-\rho_{a}||\right)^{2 k} d t \ll \sum_{n=\lfloor T\rfloor}^{\lfloor 2 T\rfloor+1} \Gamma(2 k+1)\left(\sum_{\rho_{a} \in \mathcal{D}_{n}} 1\right)^{2 k} \\
& \leq T \Gamma(2 k+1)\left(C \log M_{r+\delta}\left(s_{0}\right)\right)^{2 k}
\end{aligned}
$$

for some absolute constant $C>0$.

Lemma 5.4. Let $\frac{1}{2}<\sigma \leq 2$ be fixed. For any fixed $\sigma_{0}>1$ and $R=\sigma_{0}-\sigma$ we have

$$
\int_{T}^{2 T}\left(M_{R}\left(s_{0}\right)\right)^{2} d t \ll T .
$$

Proof. First of all,

$$
\int_{T}^{2 T}\left(M_{R}\left(s_{0}\right)\right)^{2} d t \leq \sum_{n=\lfloor T\rfloor}^{\lfloor 2 T\rfloor+1} \int_{n}^{n+1}\left(M_{R}\left(s_{0}\right)\right)^{2} d t .
$$

Next, let $D_{R}(z)$ be the disk of radius $R$ centered at $z$. Also, let $s_{n}=\sigma_{n}+i t_{n}$ be a point at which $|\zeta(s)|$ achieves its maximum value on the set $\cup_{n \leq t \leq n+1} D_{R}\left(s_{0}\right)$. Thus,

$$
\int_{n}^{n+1}\left(M_{R}\left(s_{0}\right)\right)^{2} d t \ll\left|\zeta\left(s_{n}\right)\right|^{2}+1 .
$$

Hence, we have

$$
\int_{T}^{2 T}\left(M_{R}\left(s_{0}\right)\right)^{2} d t \ll \sum_{n=\lfloor T\rfloor}^{\lfloor 2 T\rfloor+1}\left|\zeta\left(s_{n}\right)\right|^{2}+T .
$$

Let $R^{\prime}=\sigma_{0}-(\sigma+1 / 2) / 2$. To bound $\left|\zeta\left(s_{n}\right)\right|^{2}$ we note that

$$
\left|\zeta\left(s_{n}\right)\right|^{2} \leq \frac{4}{\pi\left(\sigma-\frac{1}{2}\right)^{2}} \iint_{D_{R^{\prime}}\left(\sigma_{0}+i t_{n}\right)}|\zeta(x+i y)|^{2} d x d y
$$

(For a proof of this inequality see the lemma preceding Theorem 11.9 of Titchmarsh [16]). 
Let $\mathcal{S}_{j}=\left\{s_{n}: n \equiv j\left(\bmod \left(4\left\lceil R^{\prime}\right\rceil+2\right)\right)\right\}$. If $s_{m}, s_{n} \in \mathcal{S}_{j}$ and $m \neq n$ then $|m-n| \geq$ $4\left\lceil R^{\prime}\right\rceil+2$; so that $\left|t_{m}-t_{n}\right| \geq 2 R^{\prime}+1$. This implies that $D_{R^{\prime}}\left(\sigma_{0}+i t_{n}\right) \cap D_{R^{\prime}}\left(\sigma_{0}+i t_{m}\right)=\emptyset$. Thus, since the disks are disjoint we see that by (5.9) we have

$$
\sum_{s_{n} \in S_{j}}\left|\zeta\left(s_{n}\right)\right|^{2} \ll \int_{\frac{1}{2} \cdot\left(\sigma+\frac{1}{2}\right)}^{2 \sigma_{0}-\frac{1}{2} \cdot\left(\sigma+\frac{1}{2}\right)} \int_{T-R^{\prime}-R-1}^{2 T+R^{\prime}+R+1}|\zeta(u+i t)|^{2} d t d u .
$$

Applying, the well-known mean value estimate for $\zeta(s)$ to the inner integral (see Theorem 7.2(A) of [16]) we have (uniformly in $j$ )

$$
\sum_{s_{n} \in S_{j}}\left|\zeta\left(s_{n}\right)\right|^{2} \ll T
$$

Also, $\left\{s_{n}\right\}=\coprod_{j} \mathcal{S}_{j}$. Thus,

$$
\sum_{n=\lfloor T\rfloor}^{\lfloor 2 T\rfloor+1}\left|\zeta\left(s_{n}\right)\right|^{2}=\sum_{j=0}^{4\left\lceil R^{\prime}\right\rceil+1} \sum_{s_{n} \in \mathcal{S}_{j}}\left|\zeta\left(s_{n}\right)\right|^{2} \ll T .
$$

Inserting this into (5.8) completes the proof.

Lemma 5.5. Let $\frac{1}{2}<\sigma \leq 2$ be fixed. Also, let $r, \delta$, and $\sigma_{0}$ be as in (15.2). Then, there exists an absolute constant $C>0$ such that for any real number $k \geq 1$

$$
\int_{T}^{2 T}\left(\log M_{r+\delta}\left(s_{0}\right)\right)^{2 k} d t \ll T(C k)^{2 k} .
$$

Proof. Let $f(x)=\left(\log \left(x+e^{2 k-1}\right)\right)^{2 k}$, where $k \geq 1$. Note that $f^{\prime \prime}(x)<0$ for $x>0$. Thus, by Jensen's inequality and Lemma 5.4 we have

$$
\begin{aligned}
\frac{1}{T} \int_{T}^{2 T}\left(\log M_{r+\delta}\left(s_{0}\right)\right)^{2 k} d t & <\frac{1}{4^{k} T} \int_{T}^{2 T}\left(\log \left(\left(M_{r+\delta}\left(s_{0}\right)\right)^{2}+e^{2 k-1}\right)\right)^{2 k} d t \\
& \leq \frac{1}{4^{k}}\left(\log \left(\frac{1}{T} \int_{T}^{2 T}\left(M_{r+\delta}\left(s_{0}\right)\right)^{2} d t+e^{2 k-1}\right)\right)^{2 k} \\
& \ll(C k)^{2 k},
\end{aligned}
$$

for some absolute constant $C$.

Proof of Proposition [2.5. First we consider the case $k \geq 1$. Note that by Lemma 5.2 we have

$$
\begin{aligned}
\int_{T}^{2 T}|\log | \zeta(\sigma+i t)-\left.a\right|^{2 k} d t \leq & 4^{k} \int_{T}^{2 T}\left(\sum_{\left|\rho_{a}-s_{0}\right| \leq r-\delta}|\log | \sigma+i t-\rho_{a}||\right)^{2 k} d t \\
& +O\left(4^{k} \int_{T}^{2 T}\left(\log M_{r}\left(s_{0}\right)\right)^{2 k} d t\right) .
\end{aligned}
$$


Hence, for this case, we see that Proposition 2.5 follows from the above inequality, Lemma 5.3, and Lemma 5.5. For $0<k<1$ the proposition follows from an application of Hölder's inequality.

\section{Bounding the Discrepancy: Proof of Theorem 1.1}

In order to prove Theorem 1.1 we shall appeal to the following Lemma of Selberg (Lemma 4.1 of [17]), which provides a smooth approximation for the signum function. Selberg used this lemma in his proof that $\log \zeta\left(\frac{1}{2}+i t\right)$ has a limiting two-dimensional Gaussian distribution (see [17] and [15]). Recall that the signum function is defined by

$$
\operatorname{sgn}(x)= \begin{cases}-1 & \text { if } x<0 \\ 0 & \text { if } x=0 \\ 1 & \text { if } x>0\end{cases}
$$

Lemma 6.1 (Selberg, Lemma 4.1 of [17]). Let $L>0$. Define

$$
G(u)=\frac{2 u}{\pi}+2(1-u) u \cot (\pi u) \quad \text { for } u \in[0,1] .
$$

Then for all $x \in \mathbb{R}$ we have

$$
\operatorname{sgn}(x)=\int_{0}^{L} G\left(\frac{u}{L}\right) \sin (2 \pi u x) \frac{d u}{u}+O\left(\left(\frac{\sin (\pi L x)}{\pi L x}\right)^{2}\right) .
$$

Moreover, $G(u)$ is differentiable and $0 \leq G(u) \leq 2 / \pi$ for $u \in[0,1]$.

For any rectangle $\mathcal{R}$ in the complex plane, let $\mathbf{1}_{\mathcal{R}}$ denote its indicator function. Using Lemma 6.1 we derive a smooth approximation for $\mathbf{1}_{\mathcal{R}}$ which will be used to prove Theorem 1.1. For any $\alpha, \beta \in \mathbb{R}$, we define

$$
f_{\alpha, \beta}(u):=\frac{e^{-2 \pi i \alpha u}-e^{-2 \pi i \beta u}}{2} .
$$

Then, we prove

Lemma 6.2. Let $\mathcal{R}=\left\{z=x+i y \in \mathbb{C}: a_{1}<x<a_{2}\right.$ and $\left.b_{1}<y<b_{2}\right\}$, and $L>0$ be $a$ real number. For any $z=x+i y \in \mathbb{C}$ we have

$$
\begin{aligned}
\mathbf{1}_{\mathcal{R}}(z)=W_{L, \mathcal{R}}(z)+ & O\left(\frac{\sin ^{2}\left(\pi L\left(x-a_{1}\right)\right)}{\left(\pi L\left(x-a_{1}\right)\right)^{2}}+\frac{\sin ^{2}\left(\pi L\left(x-a_{2}\right)\right)}{\left(\pi L\left(x-a_{2}\right)\right)^{2}}\right. \\
+ & \left.\frac{\sin ^{2}\left(\pi L\left(y-b_{1}\right)\right)}{\left(\pi L\left(y-b_{1}\right)\right)^{2}}+\frac{\sin ^{2}\left(\pi L\left(y-b_{2}\right)\right)}{\left(\pi L\left(y-b_{2}\right)\right)^{2}}\right)
\end{aligned}
$$

where $W_{L, \mathcal{R}}(z)$ equals

$$
\frac{1}{2} \operatorname{Re} \int_{0}^{L} \int_{0}^{L} G\left(\frac{u}{L}\right) G\left(\frac{v}{L}\right)\left(e^{2 \pi i(u x-v y)} f_{a_{1}, a_{2}}(u) \overline{f_{b_{1}, b_{2}}(v)}-e^{2 \pi i(u x+v y)} f_{a_{1}, a_{2}}(u) f_{b_{1}, b_{2}}(v)\right) \frac{d u}{u} \frac{d v}{v} .
$$


DISCREPANCY BOUNDS FOR THE DISTRIBUTION OF THE RIEMANN ZETA-FUNCTION

Proof. Here and throughout we shall denote by $\mathbf{1}_{\alpha, \beta}$ the indicator function of the interval $(\alpha, \beta)$. Observe that

$$
\mathbf{1}_{\alpha, \beta}(x)=\frac{\operatorname{sgn}(x-\alpha)-\operatorname{sgn}(x-\beta)}{2}+O(\delta(x-\alpha)+\delta(x-\beta)),
$$

where $\delta(x)$ is the Dirac delta function (it equals 1 when $x=0$, and zero otherwise).

Furthermore, it follows from Lemma 6.1 that

$$
\mathbf{1}_{\alpha, \beta}(x)=\operatorname{Im} \int_{0}^{L} G\left(\frac{u}{L}\right) e^{2 \pi i u x} f_{\alpha, \beta}(u) \frac{d u}{u}+O\left(\frac{\sin ^{2}(\pi L(x-\alpha))}{(\pi L(x-\alpha))^{2}}+\frac{\sin ^{2}(\pi L(x-\beta))}{(\pi L(x-\beta))^{2}}\right) .
$$

The result follows from the fact that $\mathbf{1}_{\mathcal{R}}(z)=\mathbf{1}_{a_{1}, a_{2}}(x) \mathbf{1}_{b_{1}, b_{2}}(y)$ together with (6.1) and the identity

$$
\operatorname{Im}\left(w_{1}\right) \operatorname{Im}\left(w_{2}\right)=\frac{1}{2} \operatorname{Re}\left(w_{1} \overline{w_{2}}-w_{1} w_{2}\right) .
$$

The last ingredient we need in order to establish Theorem 1.1 is the following lemma.

Lemma 6.3. Let $\frac{1}{2}<\sigma \leq 1$. Let $u$ be a large positive real number, then

$$
\mathbb{E}(\exp (i u \operatorname{Re} \log \zeta(\sigma, X))) \ll \exp \left(-\frac{u}{5 \log u}\right)
$$

and

$$
\mathbb{E}(\exp (i u \operatorname{Im} \log \zeta(\sigma, X))) \ll \exp \left(-\frac{u}{5 \log u}\right)
$$

Proof. First, note that $\mathbb{E}\left(e^{i s \operatorname{Re} X(p)}\right)=\mathbb{E}\left(e^{i s \operatorname{Im} X(p)}\right)=J_{0}(s)$ for all $s \in \mathbb{R}$ and all primes $p$, where $J_{0}(s)$ is the Bessel function of order 0 . We shall prove only the first inequality since the second can be derived similarly. We have

$$
\mathbb{E}(\exp (i u \operatorname{Re} \log \zeta(\sigma, X)))=\prod_{p} \mathbb{E}\left(\exp \left(-i u \operatorname{Re} \log \left(1-\frac{X(p)}{p^{\sigma}}\right)\right)\right) .
$$

Therefore, we deduce that

$$
\begin{aligned}
|\mathbb{E}(\exp (i u \operatorname{Re} \log \zeta(\sigma, X)))| & \leq \prod_{\sqrt{u} \leq p \leq u} \mathbb{E}\left(\exp \left(\frac{i u}{p^{\sigma}} \operatorname{Re} X(p)+O\left(\frac{u}{p^{2 \sigma}}\right)\right)\right) \\
& =\exp \left(O\left(u^{3 / 2-\sigma}\right)\right) \prod_{\sqrt{u} \leq p \leq u / 2} J_{0}\left(\frac{u}{p^{\sigma}}\right) .
\end{aligned}
$$

Now, using that $\left|J_{0}(x)\right| \leq e^{-1 / 2}$ for all $x \geq 2$, along with the prime number theorem we obtain

$$
|\mathbb{E}(\exp (i u \operatorname{Re} \log \zeta(\sigma, X)))| \ll \exp \left(-\frac{1}{2} \pi(u / 2)+O\left(u^{3 / 2-\sigma}\right)\right) \ll \exp \left(-\frac{u}{5 \log u}\right)
$$

as desired. 
Proof of Theorem 1.1. We only consider the case where $\frac{1}{2}<\sigma<1$, since the analogous result for $\sigma=1$ can be obtained along similar lines. To shorten our notation we let

$$
\Psi_{T}(\mathcal{R})=\mathbb{P}_{T}(\log \zeta(\sigma+i t) \in \mathcal{R}), \text { and } \Psi(\mathcal{R})=\mathbb{P}(\log \zeta(\sigma, X) \in \mathcal{R}) .
$$

Let $\mathcal{R}$ be a rectangle with sides parallel to the coordinate axes, and $\widetilde{\mathcal{R}}=\mathcal{R} \cap$ $[-\log \log T, \log \log T] \times[-\log \log T, \log \log T]$. Then using the large deviation result (1.1) we deduce that

$$
\Psi_{T}(\mathcal{R})=\Psi_{T}(\widetilde{\mathcal{R}})+O\left(\frac{1}{(\log T)^{2}}\right)
$$

Similarly, one has

$$
\Psi(\mathcal{R})=\Psi(\widetilde{\mathcal{R}})+O\left(\frac{1}{(\log T)^{2}}\right)
$$

Let $\mathcal{S}$ be the set of rectangles $\mathcal{R} \subset[-\log \log T, \log \log T] \times[-\log \log T, \log \log T]$ with sides parallel to the coordinate axes. Then, we deduce that

$$
D_{\sigma}(T)=\sup _{\mathcal{R} \in \mathcal{S}}\left|\Psi_{T}(\mathcal{R})-\Psi(\mathcal{R})\right|+O\left(\frac{1}{(\log T)^{2}}\right)
$$

Let $\mathcal{R}$ be a rectangle in $\mathcal{S}$ and $L$ a positive real number to be chosen later. Then it follows from Lemma 6.2 that

$\Psi_{T}(\mathcal{R})=\frac{1}{T} \int_{T}^{2 T} W_{L, \mathcal{R}}(\log \zeta(\sigma+i t)) d t+O\left(I_{T}\left(L, a_{1}\right)+I_{T}\left(L, a_{2}\right)+J_{T}\left(L, b_{1}\right)+J_{T}\left(t, b_{2}\right)\right)$

where

$$
I_{T}(L, s)=\frac{1}{T} \int_{T}^{2 T} \frac{\sin ^{2}(\pi L(\operatorname{Re} \log \zeta(\sigma+i t)-s))}{(\pi L(\operatorname{Re} \log \zeta(\sigma+i t)-s))^{2}} d t
$$

and

$$
J_{T}(L, s)=\frac{1}{T} \int_{T}^{2 T} \frac{\sin ^{2}(\pi L(\operatorname{Im} \log \zeta(\sigma+i t)-s))}{(\pi L(\operatorname{Im} \log \zeta(\sigma+i t)-s))^{2}} d t .
$$

It follows from Theorem 2.1 that there exists a positive constant $c=c(\sigma)$ such that for all $|u|,|v| \leq c(\log T)^{\sigma}$ we have

$$
\Phi_{\sigma, T}(u, v)=\Phi_{\sigma}^{\mathrm{rand}}(u, v)+O\left(\frac{1}{(\log T)^{5}}\right) .
$$

First, we handle the main term of (6.3)

$$
\begin{array}{r}
\frac{1}{T} \int_{T}^{2 T} W_{L, \mathcal{R}}(\log \zeta(\sigma+i t)) d t=\frac{1}{2} \operatorname{Re} \int_{0}^{L} \int_{0}^{L} G\left(\frac{u}{L}\right) G\left(\frac{v}{L}\right)\left(\Phi_{\sigma, T}(2 \pi u,-2 \pi v) f_{a_{1}, a_{2}}(u) \overline{f_{b_{1}, b_{2}}(v)}\right. \\
\left.-\Phi_{\sigma, T}(2 \pi u, 2 \pi v) f_{a_{1}, a_{2}}(u) f_{b_{1}, b_{2}}(v)\right) \frac{d u}{u} \frac{d v}{v}
\end{array}
$$


DISCREPANCY BOUNDS FOR THE DISTRIBUTION OF THE RIEMANN ZETA-FUNCTION 21

We choose $L=c(\log T)^{\sigma}$. Then inserting the estimate (6.4) in equation (6.5) and using that

$$
\left|f_{\alpha, \beta}(u)\right|=\frac{1}{2}\left|\int_{2 \pi \alpha u}^{2 \pi \beta u} e^{-i t} d t\right| \leq \pi u|\beta-\alpha|
$$

we obtain

$$
\begin{aligned}
\frac{1}{T} \int_{T}^{2 T} W_{L, \mathcal{R}}(\log \zeta(\sigma+i t)) d t & =\mathbb{E}\left(W_{L, \mathcal{R}}(\log \zeta(\sigma, X))\right)+O\left(\operatorname{meas}_{2}(\mathcal{R}) \frac{L^{2}}{(\log T)^{5}}\right) \\
& =\mathbb{E}\left(W_{L, \mathcal{R}}(\log \zeta(\sigma, X))\right)+O\left(\frac{1}{(\log T)^{2}}\right),
\end{aligned}
$$

where meas $_{2}$ denotes the two-dimensional Lebesgue measure. Furthermore we infer from Lemma 6.2

$$
\begin{aligned}
\mathbb{E}\left(W_{L, \mathcal{R}}(\log \zeta(\sigma, X))\right)= & \mathbb{E}\left(\mathbf{1}_{\mathcal{R}}(\log \zeta(\sigma, X))\right) \\
& +O\left(I_{\text {rand }}\left(L, a_{1}\right)+I_{\text {rand }}\left(L, a_{2}\right)+J_{\text {rand }}\left(L, b_{1}\right)+J_{\text {rand }}\left(L, b_{2}\right)\right),
\end{aligned}
$$

where

$$
I_{\text {rand }}(L, s)=\mathbb{E}\left(\frac{\sin ^{2}(\pi L(\operatorname{Re} \log \zeta(\sigma, X)-s))}{(\pi L(\operatorname{Re} \log \zeta(\sigma, X)-s))^{2}}\right)
$$

and

$$
J_{\text {rand }}(L, s)=\mathbb{E}\left(\frac{\sin ^{2}(\pi L(\operatorname{Im} \log \zeta(\sigma, X)-s))}{(\pi L(\operatorname{Im} \log \zeta(\sigma, X)-s))^{2}}\right) .
$$

Note that $\mathbb{E}\left(\mathbf{1}_{\mathcal{R}}(\log \zeta(\sigma, X))\right)=\mathbb{P}(\log \zeta(\sigma, X) \in \mathcal{R})$. Moreover, in order to bound $I_{\text {rand }}(L, s)$ and $J_{\text {rand }}(L, s)$ we use the following identity

$$
\frac{\sin ^{2}(\pi L x)}{(\pi L x)^{2}}=\frac{2(1-\cos (2 \pi L x))}{L^{2}(2 \pi x)^{2}}=\frac{2}{L^{2}} \int_{0}^{L}(L-v) \cos (2 \pi x v) d v .
$$

Indeed, using (6.9) along with Lemma 6.3 we obtain

$$
\begin{aligned}
I_{\text {rand }}(L, s) & =\mathbb{E}\left(\operatorname{Re} \int_{0}^{L} \frac{2(L-v)}{L^{2}} \exp (2 \pi i v(\operatorname{Re} \log \zeta(\sigma, X)-s)) d v\right) \\
& =\operatorname{Re} \int_{0}^{L} \frac{2(L-v)}{L^{2}} e^{-2 \pi i v s} \Phi_{\sigma}^{\mathrm{rand}}(2 \pi v, 0) d v \\
& \ll \frac{1}{L}\left(1+\int_{2}^{L} \exp \left(-\frac{v}{\log v}\right) d v\right) \\
& \ll \frac{1}{L} .
\end{aligned}
$$

uniformly for all $s \in \mathbb{R}$. Similarly, one obtains that $J_{\text {rand }}(L, s) \ll 1 / L$. Therefore, inserting these estimates in (6.8) and using (6.7) we deduce

$$
\frac{1}{T} \int_{T}^{2 T} W_{L, \mathcal{R}}(\log \zeta(\sigma+i t)) d t=\mathbb{P}(\log \zeta(\sigma, X) \in \mathcal{R})+O\left(\frac{1}{L}\right) .
$$


Now it remains to bound the error term on the right hand side of (6.3). Using the identity (6.9) along with equations (6.4) and (6.10) we obtain

$$
\begin{aligned}
I_{T}(L, s) & =\operatorname{Re} \frac{1}{T} \int_{T}^{2 T} \int_{0}^{L} \frac{2(L-v)}{L^{2}} \exp (2 \pi i v(\operatorname{Re} \log \zeta(\sigma+i t)-s)) d v d t \\
& =\operatorname{Re} \int_{0}^{L} \frac{2(L-v)}{L^{2}} e^{-2 \pi i v s} \Phi_{\sigma, T}(2 \pi v, 0) d v \\
& =\operatorname{Re} \int_{0}^{L} \frac{2(L-v)}{L^{2}} e^{-2 \pi i v s} \Phi_{\sigma}^{\mathrm{rand}}(2 \pi v, 0) d v+O\left(\frac{1}{(\log T)^{5}}\right) \\
& \ll \frac{1}{L},
\end{aligned}
$$

uniformly for all $s \in \mathbb{R}$. Moreover, the bound $J_{T}(L, s) \ll 1 / L$ can be obtained along the same lines. Therefore, combining these estimates with (6.3) and (6.11) we deduce

$$
\Psi_{T}(\mathcal{R})=\Psi(\mathcal{R})+O\left(\frac{1}{(\log T)^{\sigma}}\right)
$$

which completes the proof.

\section{Large Deviations: Proof of Theorem 1.2}

For $z \in \mathbb{C}$ we define

$$
M(z)=\log \mathbb{E}\left(|\zeta(\sigma, X)|^{z}\right) .
$$

Further, let $\kappa$ be the unique positive solution to the equation $M^{\prime}(k)=\tau$. One of the main ingredients in the proof of Theorem 1.2 is the following proposition which is established using the saddle-point method.

Proposition 7.1. Let $\frac{1}{2}<\sigma<1$. Uniformly for $\tau \geq 1$ we have

$$
\mathbb{P}(\log |\zeta(\sigma, X)|>\tau)=\frac{\mathbb{E}\left(|\zeta(\sigma, X)|^{\kappa}\right) e^{-\tau \kappa}}{k \sqrt{2 \pi M^{\prime \prime}(\kappa)}}\left(1+O\left(\kappa^{1-\frac{1}{\sigma}} \log \kappa\right)\right) .
$$

7.1. Preliminaries. Let $\chi(y)=1$ if $y>1$ and be equal to 0 otherwise. Then we have the following smooth analogue of Perron's formula, which is a slight variation of a formula of Granville and Soundararajan (see [4]).

Lemma 7.2. Let $\lambda>0$ be a real number and $N$ be a positive integer. For any $c>0$ we have for $y>0$

$$
0 \leq \frac{1}{2 \pi i} \int_{c-i \infty}^{c+i \infty} y^{s}\left(\frac{e^{\lambda s}-1}{\lambda s}\right)^{N} \frac{d s}{s}-\chi(y) \leq \frac{1}{2 \pi i} \int_{c-i \infty}^{c+i \infty} y^{s}\left(\frac{e^{\lambda s}-1}{\lambda s}\right)^{N} \frac{1-e^{-\lambda N s}}{s} d s .
$$

Proof. For any $y>0$ we have

$$
\frac{1}{2 \pi i} \int_{c-i \infty}^{c+i \infty} y^{s}\left(\frac{e^{\lambda s}-1}{\lambda s}\right)^{N} \frac{d s}{s}=\frac{1}{\lambda^{N}} \int_{0}^{\lambda} \cdots \int_{0}^{\lambda} \frac{1}{2 \pi i} \int_{c-i \infty}^{c+i \infty}\left(y e^{t_{1}+\cdots+t_{n}}\right)^{s} \frac{d s}{s} d t_{1} \cdots d t_{N}
$$


so that by Perron's formula we obtain

$$
\frac{1}{2 \pi i} \int_{c-i \infty}^{c+i \infty} y^{s}\left(\frac{e^{\lambda s}-1}{\lambda s}\right)^{N} \frac{d s}{s}= \begin{cases}=1 & \text { if } y \geq 1 \\ \in[0,1] & \text { if } e^{-\lambda N} \leq y<1 \\ =0 & \text { if } 0<y<e^{-\lambda N}\end{cases}
$$

Therefore we deduce that

$$
\frac{1}{2 \pi i} \int_{c-i \infty}^{c+i \infty} y^{s} e^{-\lambda N s}\left(\frac{e^{\lambda s}-1}{\lambda s}\right)^{N} \frac{d s}{s} \leq \chi(y) \leq \frac{1}{2 \pi i} \int_{c-i \infty}^{c+i \infty} y^{s}\left(\frac{e^{\lambda s}-1}{\lambda s}\right)^{N} \frac{d s}{s}
$$

which implies the result.

Lemma 7.3. Let $s=k+$ it where $k$ is a large positive real number. Then, in the range $|t| \geq k$ we have

$$
\mathbb{E}\left(|\zeta(\sigma, X)|^{s}\right) \ll \exp \left(-|t|^{1 / \sigma-1}\right) \mathbb{E}\left(|\zeta(\sigma, X)|^{k}\right) .
$$

Proof. For simplicity we suppose that $t>0$. First, note that

$$
\mathbb{E}\left(|\zeta(\sigma, X)|^{s}\right)=\prod_{p} \mathbb{E}\left(\left|1-\frac{X(p)}{p^{\sigma}}\right|^{-s}\right)
$$

Therefore, for any $y \geq 2$ we have

$$
\frac{\left|\mathbb{E}\left(|\zeta(\sigma, X)|^{s}\right)\right|}{\mathbb{E}\left(|\zeta(\sigma, X)|^{k}\right)} \leq \prod_{p>y} \frac{\left|\mathbb{E}\left(\left|1-\frac{X(p)}{p^{\sigma}}\right|^{-k-i t}\right)\right|}{\mathbb{E}\left(\left|1-\frac{X(p)}{p^{\sigma}}\right|^{-k}\right)}
$$

Moreover, for $p>|s|^{1 /(2 \sigma)}$ we have

$$
\mathbb{E}\left(\left|1-\frac{X(p)}{p^{\sigma}}\right|^{-s}\right)=\mathbb{E}\left(\left(1-2 \frac{\operatorname{Re} X(p)}{p^{\sigma}}+\frac{1}{p^{2 \sigma}}\right)^{-s / 2}\right)=I_{0}\left(\frac{s}{p^{\sigma}}\right)\left(1+O\left(\frac{|s|}{p^{2 \sigma}}\right)\right),
$$

where $I_{0}(z):=\sum_{n=0}^{\infty}(z / 2)^{2 n} / n !^{2}$ is the modified Bessel function of order 0. Let $y=t^{2 / \sigma}$. since $I_{0}(z)=1+z^{2} / 4+O\left(|z|^{4}\right)$ for $|z| \leq 1$, we deduce that for all primes $p>y$

$$
\frac{\mathbb{E}\left(\left|1-\frac{X(p)}{p^{\sigma}}\right|^{-s}\right)}{\mathbb{E}\left(\left|1-\frac{X(p)}{p^{\sigma}}\right|^{-k}\right)}=\exp \left(\frac{s^{2}-k^{2}}{4 p^{2 \sigma}}+O\left(\frac{t}{p^{2 \sigma}}+\frac{t^{4}}{p^{4 \sigma}}\right)\right) .
$$


Since $\operatorname{Re}\left(s^{2}-k^{2}\right)=-t^{2}$, it follows from the prime number theorem and equation (7.2) that

$$
\begin{aligned}
\frac{\left|\mathbb{E}\left(|\zeta(\sigma, X)|^{s}\right)\right|}{\mathbb{E}\left(|\zeta(\sigma, X)|^{k}\right)} & \leq \exp \left(-\frac{t^{2}}{4} \sum_{p>y} \frac{1}{p^{2 \sigma}}+O\left(t \sum_{p>y} \frac{1}{p^{2 \sigma}}+t^{4} \sum_{p>y} \frac{1}{p^{4 \sigma}}\right)\right) \\
& \leq \exp \left(-c(\sigma) \frac{t^{2 / \sigma-2}}{\log t}+O\left(t^{2 / \sigma-3}\right)\right),
\end{aligned}
$$

for some constant $c(\sigma)>0$. This implies the result.

Let $f(u):=\log I_{0}(u)$. Then, a classical estimate (see for example Lemma 3.1 of [8]) asserts that $f(u) \asymp u^{2}$ if $0 \leq u \leq 1$ and $f(u) \asymp u$ if $u \geq 1$. Similarly, we have the following standard estimates

Lemma 7.4. We have

$$
\begin{aligned}
f^{\prime}(u) & \asymp \begin{cases}u & \text { if } 0 \leq u \leq 1 \\
1 & \text { if } u \geq 1 .\end{cases} \\
f^{\prime \prime}(u) & \asymp \begin{cases}1 & \text { if } 0 \leq u \leq 1 \\
u^{-1} & \text { if } u \geq 1 .\end{cases} \\
f^{\prime \prime \prime}(u) & \asymp \begin{cases}u & \text { if } 0 \leq u \leq 1 \\
u^{-2} & \text { if } u \geq 1 .\end{cases}
\end{aligned}
$$

Next, we have the following proposition from which we deduce an asymptotic formula for the saddle-point $\kappa$ in terms of $\tau$.

Proposition 7.5. For large positive real numbers $k$, we have

$$
M(k)=g_{0}(\sigma) \frac{k^{1 / \sigma}}{\log k}\left(1+O\left(\frac{1}{\log k}\right)\right),
$$

where

$$
g_{0}(\sigma):=\int_{0}^{\infty} \frac{f(u)}{u^{1 / \sigma+1}} d u
$$

and

$$
M^{\prime}(k)=g_{1}(\sigma) \frac{k^{1 / \sigma-1}}{\log k}\left(1+O\left(\frac{1}{\log k}\right)\right) .
$$

where

Similarly we have

$$
g_{1}(\sigma):=\int_{0}^{\infty} \frac{f^{\prime}(u)}{u^{1 / \sigma}} d u
$$

$$
M^{\prime \prime}(k) \asymp_{\sigma} k^{1 / \sigma-2} / \log k, \text { and } M^{\prime \prime \prime}(k) \asymp_{\sigma} k^{1 / \sigma-3} / \log k .
$$


Proof. The first estimate (7.4) follows from Proposition 3.2 of [9]. The other estimates can be proved along the same lines.

Corollary 7.6. Let $\tau$ be a large real number and let $\kappa$ be the solution to $M^{\prime}(k)=\tau$. Then

$$
\kappa=g_{2}(\sigma) \tau^{\sigma /(1-\sigma)}(\log \tau)^{\sigma /(1-\sigma)}\left(1+O\left(\frac{\log \log \tau}{\log \tau}\right)\right)
$$

where

$$
g_{2}(\sigma)=\left(\frac{\sigma}{(1-\sigma) g_{1}(\sigma)}\right)^{\sigma /(1-\sigma)}
$$

Combining Proposition 7.1, Proposition 7.5 and Corollary 7.6 we recover the following result, which was obtained by the first author in [9].

Corollary 7.7. Let $\frac{1}{2}<\sigma<1$. There exists a constant $A(\sigma)>0$ such that uniformly for $\tau \geq 2$ we have

$$
\mathbb{P}(\log |\zeta(\sigma, X)|>\tau)=\exp \left(-A(\sigma) \tau^{\frac{1}{(1-\sigma)}}(\log \tau)^{\frac{\sigma}{(1-\sigma)}}(1+o(1))\right) .
$$

\subsection{Proof of Proposition 7.1 and Theorem $\mathbf{1 . 2}$.}

Proof of Proposition 7.1. Let $0<\lambda<1 /(2 \kappa)$ be a real number to be chosen later. Using Lemma 7.2 with $N=1$ we obtain

$$
\begin{aligned}
0 & \leq \frac{1}{2 \pi i} \int_{\kappa-i \infty}^{\kappa+i \infty} \mathbb{E}\left(|\zeta(\sigma, X)|^{s}\right) e^{-\tau s} \frac{e^{\lambda s}-1}{\lambda s} \frac{d s}{s}-\mathbb{P}(\log |\zeta(\sigma, X)|>\tau) \\
& \leq \frac{1}{2 \pi i} \int_{\kappa-i \infty}^{\kappa+i \infty} \mathbb{E}\left(|\zeta(\sigma, X)|^{s}\right) e^{-\tau s} \frac{\left(e^{\lambda s}-1\right)}{\lambda s} \frac{\left(1-e^{-\lambda s}\right)}{s} d s .
\end{aligned}
$$

Since $\lambda \kappa<1 / 2$ we have $\left|e^{\lambda s}-1\right| \leq 3$ and $\left|e^{-\lambda s}-1\right| \leq 2$. Therefore, using Lemma 7.3 we obtain

$$
\int_{\kappa-i \infty}^{\kappa-i \kappa}+\int_{\kappa+i \kappa}^{\kappa+i \infty} \mathbb{E}\left(|\zeta(\sigma, X)|^{s}\right) e^{-\tau s} \frac{e^{\lambda s}-1}{\lambda s} \frac{d s}{s} \ll \frac{e^{-\kappa^{1 / \sigma-1}}}{\lambda \kappa} \mathbb{E}\left(|\zeta(\sigma, X)|^{\kappa}\right) e^{-\tau \kappa}
$$

and similarly

$$
\int_{\kappa-i \infty}^{\kappa-i \kappa}+\int_{\kappa+i \kappa}^{\kappa+i \infty} \mathbb{E}\left(|\zeta(\sigma, X)|^{s}\right) e^{-\tau s} \frac{\left(e^{\lambda s}-1\right)}{\lambda s} \frac{\left(1-e^{-\lambda s}\right)}{s} d s \ll \frac{e^{-\kappa^{1 / \sigma-1}}}{\lambda \kappa} \mathbb{E}\left(|\zeta(\sigma, X)|^{\kappa}\right) e^{-\tau \kappa}
$$

Furthermore, if $|t| \leq \kappa$ then $\left|\left(1-e^{-\lambda s}\right)\left(e^{\lambda s}-1\right)\right| \ll \lambda^{2}|s|^{2}$. Hence we derive

$$
\int_{\kappa-i \kappa}^{\kappa+i \kappa} \mathbb{E}\left(|\zeta(\sigma, X)|^{s}\right) e^{-\tau s} \frac{\left(e^{\lambda s}-1\right)}{\lambda s} \frac{\left(1-e^{-\lambda s}\right)}{s} d s \ll \lambda \kappa \mathbb{E}\left(|\zeta(\sigma, X)|^{\kappa}\right) e^{-\tau \kappa}
$$


Therefore, combining this estimate with equations (7.6), (7.7) and (77.8) we deduce that

$$
\begin{aligned}
\mathbb{P}(\log |\zeta(\sigma, X)|>\tau) & -\frac{1}{2 \pi i} \int_{\kappa-i \kappa}^{\kappa+i \kappa} \mathbb{E}\left(|\zeta(\sigma, X)|^{s}\right) e^{-\tau s} \frac{e^{\lambda s}-1}{\lambda s^{2}} d s \\
& \ll\left(\lambda \kappa+\frac{e^{-\kappa^{1 / \sigma-1}}}{\lambda \kappa}\right) \mathbb{E}\left(|\zeta(\sigma, X)|^{\kappa}\right) e^{-\tau \kappa} .
\end{aligned}
$$

On the other hand, in the region $|t| \leq \kappa$ we have

$$
\log \mathbb{E}\left(|\zeta(\sigma, X)|^{\kappa+i t}\right)=\log \mathbb{E}\left(|\zeta(\sigma, X)|^{\kappa}\right)+i t M^{\prime}(\kappa)-\frac{t^{2}}{2} M^{\prime \prime}(\kappa)+O\left(M^{\prime \prime \prime}(\kappa)|t|^{3}\right) .
$$

Also, note that

$$
\frac{e^{\lambda s}-1}{\lambda s^{2}}=\frac{1}{\kappa}\left(1-i \frac{t}{\kappa}+O\left(\lambda \kappa+\frac{t^{2}}{\kappa^{2}}\right)\right) .
$$

Hence, using that $M^{\prime}(\kappa)=\tau$ we obtain

$$
\begin{aligned}
& \mathbb{E}\left(|\zeta(\sigma, X)|^{s}\right) e^{-\tau s} \frac{e^{\lambda s}-1}{\lambda s^{2}} \\
= & \frac{1}{\kappa} \mathbb{E}\left(|\zeta(\sigma, X)|^{\kappa}\right) e^{-\tau \kappa} \exp \left(-\frac{t^{2}}{2} M^{\prime \prime}(\kappa)\right)\left(1-i \frac{t}{\kappa}+O\left(\lambda \kappa+\frac{t^{2}}{\kappa^{2}}+M^{\prime \prime \prime}(\kappa)|t|^{3}\right)\right)
\end{aligned}
$$

Therefore, we obtain

$$
\begin{aligned}
& \frac{1}{2 \pi i} \int_{\kappa-i \kappa}^{\kappa+i \kappa} \mathbb{E}\left(|\zeta(\sigma, X)|^{s}\right) e^{-\tau s} \frac{e^{\lambda s}-1}{\lambda s^{2}} d s \\
= & \frac{1}{\kappa} \mathbb{E}\left(|\zeta(\sigma, X)|^{\kappa}\right) e^{-\tau \kappa} \frac{1}{2 \pi} \int_{-\kappa}^{\kappa} \exp \left(-\frac{t^{2}}{2} M^{\prime \prime}(\kappa)\right)\left(1+O\left(\lambda \kappa+\frac{t^{2}}{\kappa^{2}}+M^{\prime \prime \prime}(\kappa)|t|^{3}\right)\right) d t
\end{aligned}
$$

since the integral involving $i t / \kappa$ vanishes. Further, we have

$$
\frac{1}{2 \pi} \int_{-\kappa}^{\kappa} \exp \left(-\frac{t^{2}}{2} M^{\prime \prime}(\kappa)\right) d t=\frac{1}{\sqrt{2 \pi M^{\prime \prime}(\kappa)}}\left(1+O\left(\exp \left(-\frac{1}{2} \kappa^{2} M^{\prime \prime}(\kappa)\right)\right)\right)
$$

and

$$
\int_{-\kappa}^{\kappa}|t|^{n} \exp \left(-\frac{t^{2}}{2} M^{\prime \prime}(\kappa)\right) d t \ll \frac{1}{M^{\prime \prime}(\kappa)^{(n+1) / 2}} .
$$

Thus, using Proposition 7.5 we deduce that

$$
\begin{aligned}
& \frac{1}{2 \pi i} \int_{\kappa-i \kappa}^{\kappa+i \kappa} \mathbb{E}\left(|\zeta(\sigma, X)|^{s}\right) e^{-\tau s} \frac{e^{\lambda s}-1}{\lambda s^{2}} d s \\
= & \frac{\mathbb{E}\left(|\zeta(\sigma, X)|^{\kappa}\right) e^{-\tau \kappa}}{\kappa \sqrt{2 \pi M^{\prime \prime}(\kappa)}}\left(1+O\left(\lambda \kappa+\kappa^{1-\frac{1}{\sigma}} \log \kappa\right)\right) .
\end{aligned}
$$

Finally, it follows from Proposition 7.5 that $\kappa \sqrt{M^{\prime \prime}(\kappa)} \asymp_{\sigma} \kappa^{1 /(2 \sigma)}(\log \kappa)^{-1 / 2}$. Thus, combining the estimates (7.9) and (7.10) and choosing $\lambda=\kappa^{-3}$ completes the proof. 
Proof of Theorem 1.2. As before, $\kappa$ denotes the unique solution to $M^{\prime}(k)=\tau$. Let $N$ be a positive integer and $0<\lambda<\min \{1 /(2 \kappa), 1 / N\}$ be a real number to be chosen later.

Let $A=10, \mathcal{E}(T)$, and $b_{4}=b_{4}(\sigma, 10)$ be as in Theorem 2.2, Let $Y=\left(b_{4}(\log T)^{\sigma}\right) / 2$. Note that, if $T$ is large enough then by Corollary 7.6 we have $\kappa \leq Y$. Let $s$ be a complex number with $\operatorname{Re}(s)=\kappa$ and $|\operatorname{Im}(s)| \leq Y$. Then, it follows from Theorem 2.2 that

$$
\frac{1}{T} \int_{[T, 2 T] \backslash \mathcal{E}(T)}|\zeta(\sigma+i t)|^{s} d t=\mathbb{E}\left(|\zeta(\sigma, X)|^{s}\right)+O\left(\frac{\mathbb{E}\left(|\zeta(\sigma, X)|^{\kappa}\right)}{(\log T)^{10}}\right) .
$$

Define

$$
I(\sigma, \tau)=\frac{1}{2 \pi i} \int_{\kappa-i \infty}^{\kappa+i \infty} \mathbb{E}\left(|\zeta(\sigma, X)|^{s}\right) e^{-\tau s}\left(\frac{e^{\lambda s}-1}{\lambda s}\right)^{N} \frac{d s}{s}
$$

and

$$
J_{T}(\sigma, \tau)=\frac{1}{2 \pi i} \int_{\kappa-i \infty}^{\kappa+i \infty}\left(\frac{1}{T} \int_{[T, 2 T] \backslash \mathcal{E}(T)}|\zeta(\sigma+i t)|^{s} d t\right) e^{-\tau s}\left(\frac{e^{\lambda s}-1}{\lambda s}\right)^{N} \frac{d s}{s} .
$$

Then, using equation (7.1) we obtain

$$
\mathbb{P}(\log |\zeta(\sigma, X)|>\tau) \leq I(\sigma, \tau) \leq \mathbb{P}(\log |\zeta(\sigma, X)|>\tau-\lambda N),
$$

and

$\mathbb{P}_{T}(\log |\zeta(\sigma+i t)|>\tau)+O(\delta(T)) \leq J_{T}(\sigma, \tau) \leq \mathbb{P}_{T}(\log |\zeta(\sigma+i t)|>\tau-\lambda N)+O(\delta(T))$,

where

$$
\delta(T)=\exp \left(-c_{0}(\sigma) \frac{\log T}{\log \log T}\right),
$$

for some positive constant $c_{0}(\sigma)$, by equation (4.9).

Further, using that $\left|e^{\lambda s}-1\right| \leq 3$ we obtain

$$
\int_{\kappa-i \infty}^{\kappa-i Y}+\int_{\kappa+i Y}^{\kappa+i \infty} \mathbb{E}\left(|\zeta(\sigma, X)|^{s}\right) e^{-\tau s}\left(\frac{e^{\lambda s}-1}{\lambda s}\right)^{N} \frac{d s}{s} \ll\left(\frac{3}{\lambda Y}\right)^{N} \mathbb{E}\left(|\zeta(\sigma, X)|^{\kappa}\right) e^{-\tau \kappa}
$$

Similarly, using (7.11) we get

$$
\begin{aligned}
\int_{\kappa-i \infty}^{\kappa-i Y}+\int_{\kappa+i Y}^{\kappa+i \infty} & \left(\frac{1}{T} \int_{[T, 2 T] \backslash \mathcal{E}(T)}|\zeta(\sigma+i t)|^{s} d t\right) e^{-\tau s}\left(\frac{e^{\lambda s}-1}{\lambda s}\right)^{N} \frac{d s}{s} \\
& \ll\left(\frac{3}{\lambda Y}\right)^{N} \mathbb{E}\left(|\zeta(\sigma, X)|^{\kappa}\right) e^{-\tau \kappa} .
\end{aligned}
$$

Further, note that $\left|\left(e^{\lambda s}-1\right) / \lambda s\right| \leq 3$, which is easily seen by looking at the cases $|\lambda s| \leq 1$ and $|\lambda s|>1$. Therefore, combining equations (7.11), (7.14) and (7.15) we obtain

$$
J_{T}(\sigma, \tau)-I(\sigma, \tau) \ll \mathbb{E}\left(|\zeta(\sigma, X)|^{\kappa}\right) e^{-\tau \kappa}\left(\frac{3^{N} Y}{(\log T)^{10}}+\left(\frac{3}{\lambda Y}\right)^{N}\right) .
$$


Furthermore, it follows from Proposition 7.1 and Proposition 7.5 that

$\mathbb{P}(\log |\zeta(\sigma, X)|>\tau) \asymp_{\sigma} \frac{\sqrt{\log \kappa}}{\kappa^{1 /(2 \sigma)}} \mathbb{E}\left(|\zeta(\sigma, X)|^{\kappa}\right) e^{-\tau \kappa} \asymp_{\sigma} \frac{(\log \tau)^{(\sigma+1) /(2 \sigma)}}{\tau^{1 /(2(1-\sigma))}} \mathbb{E}\left(|\zeta(\sigma, X)|^{\kappa}\right) e^{-\tau \kappa}$.

Thus, choosing $N=[\log \log T]$ and $\lambda=e^{10} / Y$ we deduce that

$$
J_{T}(\sigma, \tau)-I(\sigma, \tau) \ll \frac{1}{(\log T)^{5}} \mathbb{P}(\log |\zeta(\sigma, X)|>\tau) .
$$

On the other hand, it follows from Corollary 7.7 that

$$
\begin{aligned}
\mathbb{P}(\log |\zeta(\sigma, X)|>\tau \pm \lambda N) & =\mathbb{P}(\log |\zeta(\sigma, X)|>\tau) \exp \left(O\left(\lambda N(\tau \log \tau)^{\frac{\sigma}{1-\sigma}}\right)\right) \\
& =\mathbb{P}(\log |\zeta(\sigma, X)|>\tau)\left(1+O\left(\frac{(\tau \log \tau)^{\frac{\sigma}{1-\sigma}} \log \log T}{(\log T)^{\sigma}}\right)\right)
\end{aligned}
$$

Combining this last estimate with (7.12), (7.13), and (7.18) we obtain

$$
\begin{aligned}
\mathbb{P}_{T}(\log |\zeta(\sigma+i t)|>\tau) & \leq J_{T}(\sigma, \tau)+O(\delta(T)) \\
& \leq I(\sigma, \tau)+O\left(\frac{\mathbb{P}(\log |\zeta(\sigma, X)|>\tau)}{(\log T)^{5}}+\delta(T)\right) \\
& \leq \mathbb{P}(\log |\zeta(\sigma, X)|>\tau)\left(1+O\left(\frac{(\tau \log \tau)^{\frac{\sigma}{1-\sigma}} \log \log T}{(\log T)^{\sigma}}\right)\right)+O(\delta(T)),
\end{aligned}
$$

and

$$
\begin{aligned}
\mathbb{P}_{T}(\log |\zeta(\sigma+i t)|>\tau) & \geq J_{T}(\sigma, \tau+\lambda N)+O(\delta(T)) \\
& \geq I(\sigma, \tau+\lambda N)+O\left(\frac{\mathbb{P}(\log |\zeta(\sigma, X)|>\tau)}{(\log T)^{5}}+\delta(T)\right) \\
& \geq \mathbb{P}(\log |\zeta(\sigma, X)|>\tau)\left(1+O\left(\frac{(\tau \log \tau)^{\frac{\sigma}{1-\sigma}} \log \log T}{(\log T)^{\sigma}}\right)\right)+O(\delta(T)) .
\end{aligned}
$$

The result follows from these estimates together with the fact that $\mathbb{P}(\log |\zeta(\sigma, X)|>$ $\tau) \gg(\delta(T))^{1 / 2}$ in our range of $\tau$, by Corollary 7.3 ,

\section{Distribution of $a$-Points: Proof of Theorem 1.3}

8.1. Preliminaries. To shorten our notation we let $\log _{2} T=\log \log T$. Let $S(T)$ be the set of points $T \leq t \leq 2 T$ such that

$$
\max \{|\log | \zeta(\sigma+i t)||,|\arg \zeta(\sigma+i t)|\}<\log _{2} T \text { and }|\log | \zeta(\sigma+i t)|-\log | a||>\delta,
$$


where $\delta=1 /(\log T)^{\sigma}$. Similarly let $F$ be the event,

$$
\max \{|\log | \zeta(\sigma, X)||,|\arg \zeta(\sigma, X)|\}<\log _{2} T \text { and }|\log | \zeta(\sigma, X)|-\log | a||>\delta \text {. }
$$

Then we have the following lemma.

Lemma 8.1. Let $\frac{1}{2}<\sigma<1$ be fixed. We have,

$$
\frac{1}{T} \int_{T}^{2 T} \log |\zeta(\sigma+i t)-a| d t=\frac{1}{T} \int_{t \in S(T)} \log |\zeta(\sigma+i t)-a| d t+O\left(\frac{\left(\log _{2} T\right)^{2}}{(\log T)^{\sigma}}\right) .
$$

and

$$
\mathbb{E}(\log |\zeta(\sigma, X)-a|)=\mathbb{E}\left(\mathbf{1}_{F} \cdot \log |\zeta(\sigma, X)-a|\right)+O\left(\frac{\left(\log _{2} T\right)^{2}}{(\log T)^{\sigma}}\right) .
$$

Proof. Note

$$
\begin{aligned}
& \left|\frac{1}{T} \int_{t \notin S(T)} \log \right| \zeta(\sigma+i t)-a|d t| \leq \\
& \leq\left(\frac{1}{T} \cdot \operatorname{meas}\{T \leq t \leq 2 T: t \notin S(T)\}\right)^{1-1 / 2 k} \cdot\left(\frac{1}{T} \int_{T}^{2 T}|\log | \zeta(\sigma+i t)-a \|^{2 k} d t\right)^{1 / 2 k} .
\end{aligned}
$$

According to Proposition 2.5,

$$
\left(\frac{1}{T} \int_{T}^{2 T}(\log |\zeta(\sigma+i t)-a|)^{2 k} d t\right)^{1 / 2 k} \ll k^{2}
$$

while by Theorem 1.1 we have

$$
\operatorname{meas}\{T \leq t \leq 2 T: t \notin S(T)\} \ll \mathbb{P}(|\log | \zeta(\sigma, X)|-\log | a||<\delta)+O\left((\log T)^{-\sigma}\right) .
$$

The probability distribution $\mathbb{P}(\log \zeta(\sigma, X) \in \cdot)$ is absolutely continuous, and therefore the above expression is $\ll \delta+(\log T)^{-\sigma} \ll(\log T)^{-\sigma}$. Choosing $k=\log _{2} T$ leads to the desired estimate

$$
\left|\int_{t \notin S(T)} \log \right| \zeta(\sigma+i t)-a|d t| \ll \frac{\left(\log _{2} T\right)^{2}}{(\log T)^{\sigma}}
$$

and hence the claim. The proof of the second statement is similar.

We let $S_{1}(T)$ be the set of points $t \in S(T)$ such that $\log |\zeta(\sigma+i t)|>\log |a|+\delta$, and $S_{2}(T)=S(T) \backslash S_{1}(T)$. Similarly, $F_{1}$ is the sub-event of $F$ where $\log |\zeta(\sigma, X)|>\log |a|+\delta$ and $F_{2}=F \backslash F_{1}$. Moreover, we define

$$
\begin{aligned}
& \Phi_{1}(u, v)=\frac{1}{T} \text { meas }\left\{t \in S_{1}(T): \log |\zeta(\sigma+i t)| \leq u \text { and } \arg \zeta(\sigma+i t) \leq v\right\} \\
& \tilde{\Phi}_{1}(u, v)=\mathbb{P}\left(F_{1} \text { and } \log |\zeta(\sigma, X)| \leq u \text { and } \arg \zeta(\sigma, X) \leq v\right) .
\end{aligned}
$$

Also, let

$$
\begin{aligned}
& \Psi(u)=\frac{1}{T} \text { meas }\left\{t \in S_{1}(T): \log |\zeta(\sigma+i t)| \leq u\right\} \\
& \tilde{\Psi}(u)=\mathbb{P}\left(F_{1} \text { and } \log |\zeta(\sigma, X)| \leq u\right)
\end{aligned}
$$


Let $g(u, v):=\log \left(e^{u+i v}-a\right)$ and $h(u, v):=\operatorname{Re}(g(u, v))$. Note that $h$ is twice differentiable in the region of $\mathbb{R}^{2}$ where $|u-\log | a||>\delta$.

We are now going to show that

$$
\int_{t \in S(T)} \log |\zeta(\sigma+i t)-a| d t \text { and } \mathbb{E}\left[\mathbf{1}_{F} \cdot \log |\zeta(\sigma, X)-a|\right]
$$

match up to a small error term. For this we will need to integrate by parts. We establish the three necessary lemmas below.

Lemma 8.2. We have

$$
\begin{aligned}
& \frac{1}{T} \int_{t \in S_{1}(T)} \log |\zeta(\sigma+i t)-a| d t \\
= & \int_{-\log _{2} T}^{\log _{2} T} \int_{\log |a|+\delta}^{\log _{2} T} \Phi_{1}(u, v) \frac{\partial^{2} h(u, v)}{\partial u \partial v} d u d v-\frac{\operatorname{meas}\left(S_{1}(T)\right)}{T} h\left(\log _{2} T, \log _{2} T\right) \\
& +\frac{1}{T} \int_{t \in S_{1}(T)}\left(h\left(\log _{2} T, \arg \zeta(\sigma+i t)\right)+h\left(\log |\zeta(\sigma+i t)|, \log _{2} T\right)\right) d t,
\end{aligned}
$$

and

$$
\begin{aligned}
& \mathbb{E}\left(\mathbf{1}_{F_{1}} \cdot \log |\zeta(\sigma, X)-a|\right) \\
= & \int_{-\log _{2} T}^{\log _{2} T} \int_{\log |a|+\delta}^{\log _{2} T} \tilde{\Phi}_{1}(u, v) \frac{\partial^{2} h(u, v)}{\partial u \partial v} d u d v-\mathbb{P}\left(F_{1}\right) h\left(\log _{2} T, \log _{2} T\right) \\
& +\mathbb{E}\left(\mathbf{1}_{F_{1}} \cdot h\left(\log _{2} T, \arg \zeta(\sigma, X)\right)\right)+\mathbb{E}\left(\mathbf{1}_{F_{1}} \cdot h\left(\log |\zeta(\sigma, X)|, \log _{2} T\right)\right) .
\end{aligned}
$$

Proof. We only prove the first identity since the second can be obtained along similar lines. We have

$$
\begin{aligned}
& \int_{-\log _{2} T}^{\log _{2} T} \int_{\log |a|+\delta}^{\log _{2} T} \Phi_{1}(u, v) \frac{\partial^{2} h(u, v)}{\partial u \partial v} d u d v \\
& =\frac{1}{T} \int_{-\log _{2} T}^{\log _{2} T} \int_{\log |a|+\delta}^{\log _{2} T} \int_{\substack{t \in S_{1}(T) \\
\log |\zeta(\sigma+i t)| \leq u \\
\arg \zeta(\sigma+i t) \leq v}} \frac{\partial^{2} h(u, v)}{\partial u \partial v} d t d u d v \\
& =\frac{1}{T} \int_{t \in S_{1}(T)} \int_{\arg \zeta(\sigma+i t)}^{\log _{2} T} \int_{\log |\zeta(\sigma+i t)|}^{\log _{2} T} \frac{\partial^{2} h(u, v)}{\partial u \partial v} d u d v d t \\
& =\frac{1}{T} \int_{t \in S_{1}(T)} \int_{\arg \zeta(\sigma+i t)}^{\log _{2} T} \frac{\partial}{\partial v} h\left(\log _{2} T, v\right)-\frac{\partial}{\partial v} h(\log |\zeta(\sigma+i t)|, v) d v d t \\
& =\frac{1}{T} \int_{t \in S_{1}(T)} \log |\zeta(\sigma+i t)-a| d t+\frac{1}{T} \int_{t \in S_{1}(T)} h\left(\log _{2} T, \log _{2} T\right) d t \\
& -\frac{1}{T} \int_{t \in S_{1}(T)}\left(h\left(\log _{2} T, \arg \zeta(\sigma+i t)\right)+h\left(\log |\zeta(\sigma+i t)|, \log _{2} T\right)\right) d t .
\end{aligned}
$$


Lemma 8.3. Let $\frac{1}{2}<\sigma<1$ be fixed. We have

$\frac{1}{T} \int_{t \in S_{1}(T)} h\left(\log |\zeta(\sigma+i t)|, \log _{2} T\right) d t=\mathbb{E}\left(\mathbf{1}_{F_{1}} \cdot h\left(\log |\zeta(\sigma, X)|, \log _{2} T\right)\right)+O\left(\frac{\log _{2} T}{(\log T)^{\sigma}}\right)$,

and

$\frac{1}{T} \int_{t \in S_{1}(T)} h\left(\log _{2} T, \arg \zeta(\sigma+i t)\right) d t=\mathbb{E}\left(\mathbf{1}_{F_{1}} \cdot h\left(\log _{2} T, \arg \zeta(\sigma, X)\right)\right)+O\left(\frac{\log _{2} T}{(\log T)^{\sigma}}\right)$.

Proof. We only prove the first estimate since the second is similar. We have

$$
\frac{1}{T} \int_{t \in S_{1}(T)} h\left(\log |\zeta(\sigma+i t)|, \log _{2} T\right) d t=\int_{\log |a|+\delta}^{\log _{2} T} h\left(u, \log _{2} T\right) d \Psi(u) .
$$

Integrating by parts, the right-hand side equals

$$
\begin{aligned}
{\left[\Psi(u) h\left(u, \log _{2} T\right)\right]_{\log |a|+\delta}^{\log _{2} T}-\int_{\log |a|+\delta}^{\log _{2} T} h^{\prime}\left(u, \log _{2} T\right) \Psi(u) d u } \\
\quad=\left[\tilde{\Psi}(u) h\left(u, \log _{2} T\right)\right]_{\log |a|+\delta}^{\log _{2} T}-\int_{\log |a|+\delta}^{\log _{2} T} h^{\prime}\left(u, \log _{2} T\right) \tilde{\Psi}(u) d u+E_{5} \\
=\mathbb{E}\left(\mathbf{1}_{F_{1}} \cdot h\left(\log |\zeta(\sigma, X)|, \log _{2} T\right)\right)+E_{5}
\end{aligned}
$$

where

$$
E_{5} \ll \frac{1}{(\log T)^{\sigma}}\left(\log _{2} T+\int_{\log |a|+\delta}^{\log _{2} T}\left|h^{\prime}\left(u, \log _{2} T\right)\right| d u\right),
$$

which follows from the discrepancy estimate $\Psi(u)-\tilde{\Psi}(u) \ll(\log T)^{-\sigma}$, along with the bounds $h\left(\log _{2} T, \log _{2} T\right) \ll \log _{2} T$ and $h\left(\log |a|+\delta, \log _{2} T\right) \ll \log (1 / \delta) \ll \log _{2} T$. Now, we have

$$
\left|h^{\prime}\left(u, \log _{2} T\right)\right|=\left|\operatorname{Re}\left(g^{\prime}\left(u, \log _{2} T\right)\right)\right| \leq\left|g^{\prime}\left(u, \log _{2} T\right)\right| \leq \frac{e^{u}}{\left|e^{u}-\right| a||} .
$$

Further, by making the change of variable $x=u-\log |a|$, we get

$$
\int_{\log |a|+\delta}^{\log _{2} T}\left|h^{\prime}\left(u, \log _{2} T\right)\right| d u \ll \int_{\delta}^{2 \log _{2} T} \frac{e^{x}}{e^{x}-1} d x \ll \int_{\delta}^{1} \frac{d x}{x}+\log _{2} T \ll \log _{2} T .
$$

Inserting this estimate in (8.1) completes the proof.

Lemma 8.4. Let $\frac{1}{2}<\sigma<1$ be fixed. We have

$$
\begin{aligned}
\int_{-\log _{2} T}^{\log _{2} T} \int_{\log |a|+\delta}^{\log _{2} T} \Phi_{1}(u, v) \frac{\partial^{2} h(u, v)}{\partial u \partial v} d u d v= & \int_{-\log _{2} T}^{\log _{2} T} \int_{\log |a|+\delta}^{\log _{2} T} \tilde{\Phi}_{1}(u, v) \frac{\partial^{2} h(u, v)}{\partial u \partial v} d u d v \\
& +O\left(\frac{\left(\log _{2} T\right)^{2}}{(\log T)^{\sigma}}\right) .
\end{aligned}
$$


Proof. By the discrepancy estimate $\Phi_{1}(u, v)-\tilde{\Phi}_{1}(u, v) \ll(\log T)^{-\sigma}$, we obtain that

$$
\begin{aligned}
\int_{-\log _{2} T}^{\log _{2} T} \int_{\log |a|+\delta}^{\log _{2} T} \Phi_{1}(u, v) \frac{\partial^{2} h(u, v)}{\partial u \partial v} d u d v= & \int_{-\log _{2} T}^{\log _{2} T} \int_{\log |a|+\delta}^{\log _{2} T} \tilde{\Phi}_{1}(u, v) \frac{\partial^{2} h(u, v)}{\partial u \partial v} d u d v \\
& +O\left(\frac{1}{(\log T)^{\sigma}} \int_{\log |a|+\delta}^{\log _{2} T} \int_{-\log _{2} T}^{\log _{2} T}\left|\frac{\partial^{2} h(u, v)}{\partial u \partial v}\right| d v d u\right) .
\end{aligned}
$$

Note that

$$
\left|\frac{\partial^{2} h(u, v)}{\partial u \partial v}\right|=\left|\operatorname{Re} \frac{\partial^{2} g(u, v)}{\partial u \partial v}\right| \leq\left|\frac{\partial^{2} g(u, v)}{\partial u \partial v}\right| \ll \frac{e^{u}}{\left|e^{u+i v}-a\right|^{2}},
$$

and $\left|e^{u+i v}-a\right|^{2}=e^{2 u}+|a|^{2}-2 \operatorname{Re}\left(a e^{u-i v}\right)=\left(e^{u}-|a|\right)^{2}+2|a| e^{u}(1-\cos (v-\arg a))$. We split the range of integration over $v$ into intervals $[-\pi+2 \pi k+\arg a, \pi+2 \pi k+\arg a]$ with $|k| \leq\left(\log _{2} T\right) / \pi$. Since the integrand is non-negative, we deduce that

$$
\begin{aligned}
\int_{-\log _{2} T}^{\log _{2} T}\left|\frac{\partial^{2} h(u, v)}{\partial u \partial v}\right| d v & \leq e^{u} \sum_{|k| \leq \log _{2} T} \int_{-\pi+2 \pi k+\arg a}^{\pi+2 \pi k+\arg a} \frac{1}{\left(e^{u}-|a|\right)^{2}+2|a| e^{u}(1-\cos (v-\arg a))} d v \\
& \ll e^{u} \log _{2} T \int_{0}^{\pi} \frac{1}{\left(e^{u}-|a|\right)^{2}+2|a| e^{u}(1-\cos v)} d v,
\end{aligned}
$$

by a simple change of variable and since the integrand is an even function of $v$. Furthermore, using that $1-\cos v \geq v^{2} / 10$ for $0 \leq v \leq \pi$ we obtain that

$$
\int_{0}^{\pi} \frac{1}{\left(e^{u}-|a|\right)^{2}+2|a| e^{u}(1-\cos v)} d v \leq \int_{0}^{\pi} \frac{1}{\left(e^{u}-|a|\right)^{2}+|a| e^{u} v^{2} / 5} d v .
$$

Now, by making the change of variable

$$
y=\frac{\sqrt{|a|} e^{u / 2}}{\sqrt{5}\left(e^{u}-|a|\right)} v
$$

we derive

$$
\int_{0}^{\pi} \frac{1}{\left(e^{u}-|a|\right)^{2}+|a| e^{u} v^{2} / 5} d v \ll \frac{e^{-u / 2}}{e^{u}-|a|} \int_{0}^{\infty} \frac{1}{1+y^{2}} d y \ll \frac{e^{-u / 2}}{e^{u}-|a|} .
$$

Combining these estimates we deduce that

$$
\int_{\log |a|+\delta}^{\log _{2} T} \int_{-\log _{2} T}^{\log _{2} T}\left|\frac{\partial^{2} h(u, v)}{\partial u \partial v}\right| d v d u \ll \log _{2} T \int_{\log |a|+\delta}^{\log _{2} T} \frac{e^{u / 2}}{e^{u}-|a|} d u \ll \log _{2} T \int_{\delta}^{2 \log _{2} T} \frac{e^{x / 2}}{e^{x}-1} d x
$$

by making the change of variable $x=u-\log |a|$ and since the integrand is positive. The lemma follows upon noting that

$$
\int_{\delta}^{2 \log _{2} T} \frac{e^{x / 2}}{e^{x}-1} d x \ll \int_{\delta}^{1} \frac{1}{x} d x+\int_{1}^{\log _{2} T} e^{-x / 2} d x \ll \log _{2} T .
$$


DISCREPANCY BOUNDS FOR THE DISTRIBUTION OF THE RIEMANN ZETA-FUNCTION 33

\subsection{Proofs of Theorems 2.4 and 1.3 .}

Proof of Theorem 2.4. In view of Lemma 8.1 we only need to prove that

$$
\frac{1}{T} \int_{t \in S(T)} \log |\zeta(\sigma+i t)-a| d t=\mathbb{E}\left(\mathbf{1}_{F} \cdot \log |\zeta(\sigma, X)-a|\right)+O\left(\frac{\left(\log _{2} T\right)^{2}}{(\log T)^{\sigma}}\right) .
$$

Recall that $S(T)=S_{1}(T) \cup S_{2}(T)$ and $F=F_{1} \cup F_{2}$. Combining the discrepancy estimate

$$
\frac{\operatorname{meas}\left(S_{1}(T)\right)}{T}-\mathbb{P}\left(F_{1}\right) \ll(\log T)^{-\sigma}
$$

with Lemmas 8.2 , 8.3 and 8.4, we obtain

$$
\frac{1}{T} \int_{t \in S_{1}(T)} \log |\zeta(\sigma+i t)-a| d t=\mathbb{E}\left(\mathbf{1}_{F_{1}} \cdot \log |\zeta(\sigma, X)-a|\right)+O\left(\frac{\left(\log _{2} T\right)^{2}}{(\log T)^{\sigma}}\right) .
$$

Finally, using a similar approach one obtains

$$
\frac{1}{T} \int_{t \in S_{2}(T)} \log |\zeta(\sigma+i t)-a| d t=\mathbb{E}\left(\mathbf{1}_{F_{2}} \cdot \log |\zeta(\sigma, X)-a|\right)+O\left(\frac{\left(\log _{2} T\right)^{2}}{(\log T)^{\sigma}}\right)
$$

where instead of Lemma 8.2 we use

$$
\begin{aligned}
& \frac{1}{T} \int_{t \in S_{2}(T)} \log |\zeta(\sigma+i t)-a| d t \\
= & \int_{-\log _{2} T}^{\log _{2} T} \int_{-\log _{2} T}^{\log |a|-\delta} \Phi_{2}(u, v) \frac{\partial^{2} h(u, v)}{\partial u \partial v} d u d v-\frac{\operatorname{meas}\left(S_{2}(T)\right)}{T} h\left(-\log _{2} T,-\log _{2} T\right) \\
& +\frac{1}{T} \int_{t \in S_{2}(T)}\left(h\left(-\log _{2} T, \arg \zeta(\sigma+i t)\right)+h\left(\log |\zeta(\sigma+i t)|,-\log _{2} T\right)\right) d t,
\end{aligned}
$$

with

$$
\Phi_{2}(u, v)=\frac{1}{T} \operatorname{meas}\left\{t \in S_{2}(T): \log |\zeta(\sigma+i t)| \geq u \text { and } \arg \zeta(\sigma+i t) \geq v\right\} .
$$

For the proof of Theorem 1.3 we need an auxiliary lemma.

Lemma 8.5. Let $a \neq 0$. The function

$$
f_{a}(\sigma):=\mathbb{E}[\log |\zeta(\sigma, X)-a|]
$$

is twice differentiable in $\sigma$ for $\frac{1}{2}<\sigma<1$.

Proof. See Theorem 14 of [3]. 
Proof of Theorem 1.3. Let $\frac{1}{2}<\sigma<1$ and $\rho_{a}=\beta_{a}+i \gamma_{a}$ denote an $a$-point of $\zeta(s)$. We know that there is $\sigma_{0}=\sigma_{0}(a)$ such that $\beta_{a}<\sigma_{0}$ for all $a$-points $\rho_{a}$. By Littlewood's lemma (see equation (9.9.1) of Titchmarsh [16]), we have

$$
\begin{aligned}
\int_{\sigma}^{\sigma_{0}}\left(\sum_{\substack{\beta_{a}>u \\
T \leq \gamma_{a} \leq 2 T}} 1\right) d u= & \frac{1}{2 \pi} \int_{T}^{2 T} \log |\zeta(\sigma+i t)-a| d t-\frac{1}{2 \pi} \int_{T}^{2 T} \log \left|\zeta\left(\sigma_{0}+i t\right)-a\right| d t \\
& +\frac{1}{2 \pi} \int_{\sigma}^{\sigma_{0}}(\arg (\zeta(\alpha+2 i T)-a)-\arg (\zeta(\alpha+i T)-a)) d \alpha
\end{aligned}
$$

Furthermore, a standard application of the argument principle shows that (see for example equation (8.4) of Tsang's Thesis [17])

$$
\int_{\sigma}^{\sigma_{0}}(\arg (\zeta(\alpha+2 i T)-a)-\arg (\zeta(\alpha+i T)-a)) d \alpha \ll_{a} \log T .
$$

Let $0<h<\min \left(\sigma-\frac{1}{2}, 1-\sigma\right)$. Inserting this last estimate in equation (8.2) and using Theorem 2.4 we obtain

$$
\int_{\sigma}^{\sigma+h}\left(\sum_{\substack{\beta_{a}>u \\ T \leq \gamma_{a} \leq 2 T}} 1\right) d u=\frac{T}{2 \pi} \cdot(\mathbb{E}[\log |\zeta(\sigma, X)-a|]-\mathbb{E}[\log |\zeta(\sigma+h, X)-a|])+O\left(\frac{T\left(\log _{2} T\right)^{2}}{(\log T)^{\sigma}}\right) .
$$

Recall that $f_{a}(\sigma)=\mathbb{E}[\log |\zeta(\sigma, X)-a|]$ is twice differentiable in $\sigma$ by Lemma 8.5. Hence, we derive

$$
\begin{aligned}
\frac{1}{h} \int_{\sigma}^{\sigma+h}\left(\sum_{\substack{\beta_{a}>u \\
T \leq \gamma_{a} \leq 2 T}} 1\right) d u & =\frac{T}{2 \pi} \cdot\left(\frac{f(\sigma)-f(\sigma+h)}{h}\right)+O\left(\frac{T\left(\log _{2} T\right)^{2}}{(\log T)^{\sigma}} \cdot \frac{1}{h}\right) \\
& =-\frac{T}{2 \pi} \cdot f^{\prime}(\sigma)+O\left(h T+\frac{T\left(\log _{2} T\right)^{2}}{(\log T)^{\sigma}} \cdot \frac{1}{h}\right) .
\end{aligned}
$$

Therefore,

$$
\sum_{\substack{\beta_{a} \geq \sigma+h \\ T \leq \gamma_{a} \leq 2 T}} 1 \leq-\frac{T}{2 \pi} \cdot f^{\prime}(\sigma)+O\left(h T+\frac{T\left(\log _{2} T\right)^{2}}{(\log T)^{\sigma}} \cdot \frac{1}{h}\right) \leq \sum_{\substack{\beta_{a} \geq \sigma \\ T \leq \gamma_{a} \leq 2 T}} 1
$$

We substitute $\sigma-h$ for $\sigma$ and use $f^{\prime}(\sigma-h)=f^{\prime}(\sigma)+O(h)$ to conclude that also

$$
\sum_{\substack{\beta_{a} \geq \sigma \\ T \leq \gamma_{a} \leq 2 T}} 1 \leq-\frac{T}{2 \pi} \cdot f^{\prime}(\sigma)+O\left(h T+\frac{T\left(\log _{2} T\right)^{2}}{(\log T)^{\sigma}} \cdot \frac{1}{h}\right)
$$

We pick $h=\left(\log _{2} T\right) \cdot(\log T)^{-\sigma / 2}$ to conclude that

$$
\sum_{\substack{\beta_{a} \geq \sigma \\ T \leq \gamma_{a} \leq 2 T}} 1=-\frac{T}{2 \pi} \cdot f^{\prime}(\sigma)+O\left(\frac{T \log _{2} T}{(\log T)^{\sigma / 2}}\right) .
$$

From this the claim follows. 


\section{APPENDIX: LOWER BOUNDS FOR THE DISCREPANCY}

According to [7],

$$
\int_{T}^{2 T}|\zeta(\sigma+i t)|^{2} d t=\zeta(2 \sigma) T+(2 \pi)^{2 \sigma-1} \cdot \frac{\zeta(2-2 \sigma)}{2-2 \sigma} \cdot\left(2^{2-2 \sigma}-1\right) T^{2-2 \sigma}+O\left(T^{1-\sigma}\right) .
$$

We notice that

$$
\mathbb{E}\left(|\zeta(\sigma, X)|^{2}\right)=\zeta(2 \sigma)
$$

Therefore, if $D_{\sigma}(T)=O\left(T^{1-2 \sigma-\delta}\right)$ for some $\delta>0$, then by integration by parts

$$
\int_{T}^{2 T}|\zeta(\sigma+i t)|^{2} d t=\zeta(2 \sigma) T+O\left(T^{2-2 \sigma-\delta}\right)
$$

which contradicts the previous equation. Therefore $D_{\sigma}(T)=\Omega\left(T^{1-2 \sigma-\varepsilon}\right)$. We notice that the term $T^{2-2 \sigma}$ arises from the $\chi$ factors in the approximate functional equation. Therefore the observed discrepancy $D_{\sigma}(T)=\Omega\left(T^{1-2 \sigma-\varepsilon}\right)$ ultimately arises because the probabilistic model $\zeta(\sigma, X)$ does not take into account the $\chi$ factors in the approximate functional equation (or equivalently because independence is ruined for the harmonics $n^{i t}$ and $m^{i t}$ with $n, m$ close to $\left.T\right)$.

As to the second assertion, if we have that $D_{\sigma}(T)=O\left(T^{1-2 \sigma+\varepsilon}\right)$, then again an integration by parts shows that

$$
\int_{T}^{2 T} \log |\zeta(\sigma+i t)| d t=T \cdot \mathbb{E}[\log |\zeta(\sigma, X)|]+O\left(T^{2-2 \sigma+\varepsilon}\right) .
$$

Since $\log |\zeta(\sigma, X)|$ is symmetric we have $\mathbb{E}[\log |\zeta(\sigma, X)|]=0$. By Littlewood's lemma we conclude that

$$
\sum_{\substack{\beta>\sigma \\ T \leq \gamma \leq 2 T}}(\beta-\sigma)=O\left(T^{2-2 \sigma+\varepsilon}\right) .
$$

From this it follows that the number of zeros of $\zeta(s)$ in the region $\beta>\sigma+\varepsilon$ is $\ll T^{2-2 \sigma+\varepsilon}$ as desired.

\section{ACKNOWLEDGMENTS}

We would like to thank Yoonbok Lee for discussions regarding the generalization of our result to Epstein zeta-functions and linear combinations of $L$-functions.

\section{REFERENCES}

1. Harald Bohr and Börge Jessen, Über die Werteverteilung der Riemannschen Zetafunktion, erste Mitteilung, Acta Math. 54 (1930), no. 1, 1-35. MR 1555301

2. Enrico Bombieri and Julia Mueller, On the zeros of certain Epstein zeta functions, Forum Math. 20 (2008), no. 2, 359-385. MR 2395968 (2009a:11088)

3. Vibeke Borchsenius and Børge Jessen, Mean motions and values of the Riemann zeta function, Acta Math. 80 (1948), 97-166. MR 0027796 (10,356b)

4. Andrew Granville and K. Soundararajan, Extreme values of $|\zeta(1+i t)|$, The Riemann zeta function and related themes: papers in honour of Professor K. Ramachandra, Ramanujan Math. Soc. Lect. Notes Ser., vol. 2, Ramanujan Math. Soc., Mysore, 2006, pp. 65-80. MR 2335187 (2008f:11091) 
5. Charng Rang Guo, On the zeros of the derivative of the Riemann zeta function, Proc. London Math. Soc. (3) 72 (1996), no. 1, 28-62. MR 1357088 (96i:11096)

6. Glyn Harman and Kohji Matsumoto, Discrepancy estimates for the value-distribution of the Riemann zeta-function. IV, J. London Math. Soc. (2) 50 (1994), no. 1, 17-24. MR 1277751 (95e:11090)

7. A. Ivić, Lectures on mean values of the Riemann zeta function, Tata Institute of Fundamental Research Lectures on Mathematics and Physics, vol. 82, Published for the Tata Institute of Fundamental Research, Bombay, 1991. MR 1230387 (94g:11061)

8. Youness Lamzouri, Extreme values of $\arg L(1, \chi)$, Acta Arith. 146 (2011), no. 4, 335-354. MR 2747035 (2011m:11176)

9. $\quad$ On the distribution of extreme values of zeta and L-functions in the strip $\frac{1}{2}<\sigma<1$, Int. Math. Res. Not. IMRN (2011), no. 23, 5449-5503. MR 2855075 (2012m:11113)

10. Edmund Landau, Über die Wurzeln der Zetafunktion, Math. Z. 20 (1924), no. 1, 98-104. MR 1544664

11. Yoonbok Lee, On the zeros of epstein zeta functions off the critical line, Forum. Math. to appear (2013).

12. K. Matsumoto, Discrepancy estimates for the value-distribution of the Riemann zeta-function. II, Number theory and combinatorics. Japan 1984 (Tokyo, Okayama and Kyoto, 1984), World Sci. Publishing, Singapore, 1985, pp. 265-278. MR 827789 (88i:11057)

13. Kohji Matsumoto, Discrepancy estimates for the value-distribution of the Riemann zeta-function. III, Acta Arith. 50 (1988), no. 4, 315-337. MR 961691 (90a:11102)

14. H. L. Montgomery, Extreme values of the riemann zeta function, Comment. Math. Helv. 52 (1977), no. $4,511-518$.

15. Atle Selberg, Old and new conjectures and results about a class of Dirichlet series, Proceedings of the Amalfi Conference on Analytic Number Theory (Maiori, 1989) (Salerno), Univ. Salerno, 1992, pp. 367-385. MR 1220477 (94f:11085)

16. E. C. Titchmarsh, The theory of the Riemann zeta-function, second ed., The Clarendon Press Oxford University Press, New York, 1986, Edited and with a preface by D. R. Heath-Brown. MR 882550 (88c:11049)

17. Kai-Man Tsang, THE DISTRIBUTION OF THE VALUES OF THE RIEMANN ZETAFUNCTION, ProQuest LLC, Ann Arbor, MI, 1984, Thesis (Ph.D.)-Princeton University. MR 2633927

Department of Mathematics and Statistics, York University, 4700 Keele Street, Toronto, ON M3J 1P3, CANADA

E-mail address: lamzouri@mathstat.yorku.ca

School of Mathematical Sciences, Tel Aviv University, Tel Aviv 69978, Israel

E-mail address: slester@post.tau.ac.il

School of Mathematics, Institute for Advanced Study, 1 Einstein Drive, Princeton, NJ 08540 USA

E-mail address: maksym@ias.edu 